Notes ouest-africaines

\title{
Urbanisation et démographie en Afrique du Nord et de l'Ouest, 1950-2020
}

NOVEMBRE 2021 N$^{\circ} 33$

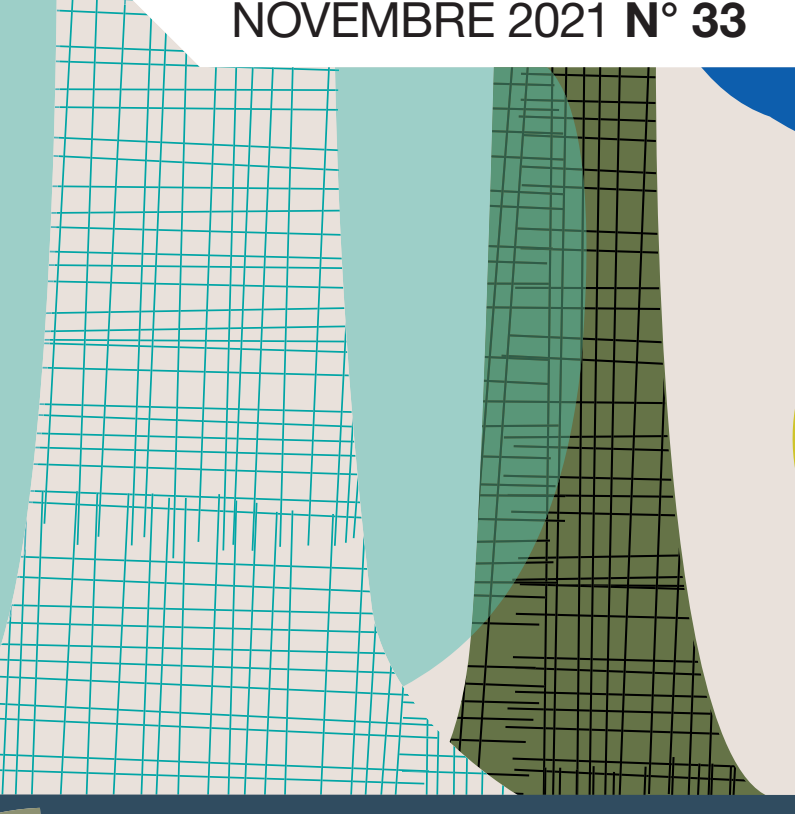

Secrétariat du

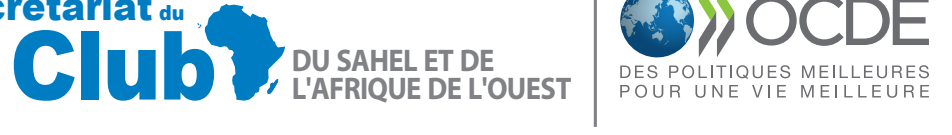




\section{URBANISATION ET DÉMOGRAPHIE EN AFRIQUE DU NORD ET DE L'OUEST, 1950-2020}

Cette note a été rédigée par

Olivier J. Walther

Groupe de recherche sur le Sahel, Université de Floride

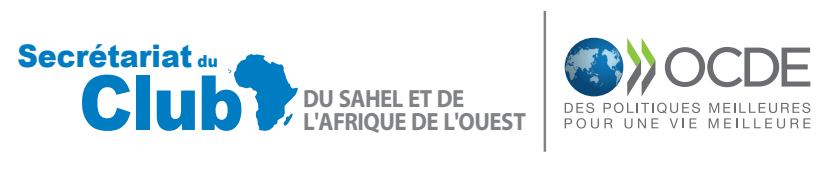




\section{NOTES OUEST-AFRICAINES}

Les Notes ouest-africaines analysent les dynamiques socio-économiques, politiques et sécuritaires que traverse l'Afrique dans une perspective régionale et multidisciplinaire. Elles cherchent à stimuler la discussion, rassembler les informations et mieux anticiper les transformations en cours pour les politiques à venir. Elles visent à partager des études avec une large audience d'experts, de praticiens du développement, de décisionnaires et de lecteurs avertis. Les Notes sont disponibles en anglais et/ou en français; les résumés dans les deux langues. Initiées par le Club du Sahel et de l'Afrique de l'Ouest (CSAO) pour éclairer les enjeux ouest-africains, ces analyses sont préparées par son Secrétariat, ses membres et partenaires, les autres départements de l'OCDE, des organisations internationales et autres experts et chercheurs.

Merci de citer cet ouvrage comme suit :

Walther, O. (2021), « Urbanisation et démographie en Afrique du Nord et de l'Ouest, 1950-2020 ), Notes ouest-africaines, N³3, Éditions OCDE, Paris.

https://doi.org/10.1787/702cc781-fr

Contact auteur : owalther@ufl.edu

Notes ouest-africaines

ISSN 2414-2026

Cet ouvrage est publié sous la responsabilité du Secrétaire général de l'OCDE. Les opinions et les arguments exprimés ici ne reflètent pas nécessairement les vues officielles des pays membres de l'OCDE.

Ce document, ainsi que les données et cartes qu'il peut comprendre, sont sans préjudice du statut de tout territoire, de la souveraineté s'exerçant sur ce dernier, du tracé des frontières et limites internationales, et du nom de tout territoire, ville ou région.

Autorisé pour publication par Laurent Bossard, Directeur, Secrétariat du Club du Sahel et de l'Afrique de l'Ouest (OCDE/CSAO).

Crédits photo : Couverture (C) Delphine Chedru. 


\section{Résumé}

Cette note propose une analyse factuelle et rétrospective des relations entre urbanisation et démographie en Afrique de l'Ouest et du Nord. Elle montre que les pays de la région sont résolument engagés dans le processus de transition démographique. Au nord du Sahara, le nouvel équilibre démographique est marqué par une natalité plus forte que ce que le modèle théorique prédit, avec pour conséquence une croissance continue de la population. Plus de $70 \%$ de la population vit désormais en ville et cette proportion devrait continuer à croître dans les prochaines décennies. Au sud du Sahara, tous les pays ont connu une chute rapide de la mortalité suivie d'un recul de la natalité. Le décalage entre l'évolution des deux variables a contribué à un accroissement naturel spectaculaire en l'espace de quelques décennies. Cette croissance s'accompagne d'une redistribution des populations en faveur des zones urbaines, qui accueillent désormais près d'un habitant sur deux. L'urbanisation que connaît l'Afrique de l'Ouest est susceptible d'accélérer les changements sociaux, économiques et politiques qui favorisent la transition démographique. L'un des défis principaux est de parvenir à réduire les variations régionales observées dans les taux de fertilité, qui s'expliquent par un délai temporel entre zones urbaines et rurales du continent.

Mots clés : démographie, population, natalité, urbanisation, Afrique de l'Ouest Codes JEL : N37, N97, Q56

\section{À propos de l'auteur}

Olivier J. Walther est professeur assistant au Département de Géographie de l'Université de Floride aux États-Unis. Titulaire d'un doctorat en géographie de l'Université de Lausanne, Dr Walther oriente ses recherches sur le commerce et les conflits en Afrique de l'Ouest en utilisant l'analyse des réseaux sociaux. Ses travaux ont été financés par la NASA, les Nations Unies, la National Science Foundation, l'OCDE et l'Union européenne. Au sein du Groupe de recherche sur le Sahel de l'Université de Floride, il coordonne des recherches sur l'insécurité régionale pour le Club du Sahel et de l'Afrique de l'Ouest (CSAO/OCDE).

\section{Note aux lecteurs}

Cette publication a reçu des fonds supplémentaires de la Direction du développement et de la coopération (DDC) suisse. 


\section{Le Club du Sahel et de l'Afrique de l'Ouest}

Le Club du Sahel et de l'Afrique de l'Ouest (CSAO) est une plateforme internationale indépendante. Son Secrétariat est hébergé au sein de l'Organisation de coopération et de développement économiques (OCDE). Sa mission est de promouvoir des politiques régionales à même d'améliorer le bien-être économique et social des populations du Sahel et de l'Afrique de l'Ouest. À cette fin, il se fixe pour objectifs de produire et de collecter des données, de fournir des analyses et de faciliter le dialogue stratégique, dans le but de favoriser et de promouvoir des politiques publiques en phase avec les transformations rapides à l'œuvre dans la région. Il promeut, en outre, la coopération régionale comme vecteur de développement durable et de stabilité. Ses domaines de travail portent actuellement sur les dynamiques alimentaires, les villes et territoires, et la sécurité.

Ses Membres et partenaires sont l'Autriche, la Belgique, le Canada, le CILSS (Comité permanent inter-États de lutte contre la sécheresse dans le Sahel), la Commission de la CEDEAO (Communauté économique des États de l'Afrique de l'Ouest), la Commission de l'UEMOA (Union économique et monétaire ouest-africaine), la Commission européenne, les États-Unis, la France, le Luxembourg, la Norvège, les Pays-Bas et la Suisse. Il a par ailleurs conclu un protocole d'accord (Memorandum of Understanding [MOU]) avec le Groupe de recherche sur le Sahel de l'Université de Floride.

Pour en savoir plus, consulter :

www.oecd.org/csao 


\section{Table des matières}

$\begin{array}{ll}\text { INTRODUCTION } & 7\end{array}$

LA TRANSITION DÉMOGRAPHIQUE $\quad 8$

CHANGEMENTS DÉMOGRAPHIQUES EN AFRIQUE DU NORD ET DE L'OUEST _............. 10

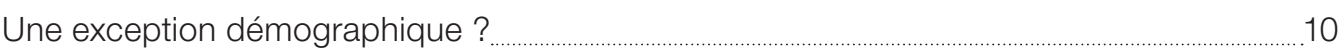

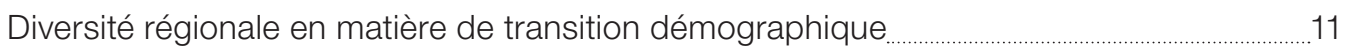

Convergence des taux de mortalité et natalité en Afrique du Nord et de l'Ouest

Croissances démographiques nationales $\quad . .15$

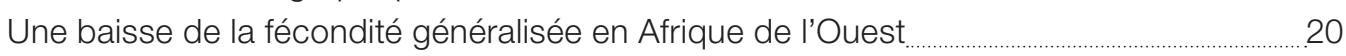

DÉMOGRAPHIE ET URBANISATION 22

Transition démographique et urbanisation vont de pair $\quad 22$

Essor de l'urbanisation _ 23

Changements à l'horizon $2050 \quad 26$

PERSPECTIVES 28

RÉFÉRENCES $\quad 29$

Graphique 1 Phases théoriques de la transition démographique _ 8

Graphique 2 Phases de la transition démographique en Afrique du Nord et de l'Ouest .....................12

Graphique 3 Transition démographique en Afrique du Nord et au Sahel, 1950-2020 _........................13

Graphique 4 Convergence de la natalité et mortalité en Afrique du Nord et Cabo Verde, 1960-2020 14

Graphique 5 Convergence de la natalité et mortalité au Sahel, 1960-2020 _.......................................15

Graphique 6 Population par pays, $2020 \quad 16$

Graphique 7 Croissance démographique et population en Afrique du Nord et au Sahel, 1950-2020 17

Graphique 8 Taux de croissance démographique par pays, 1950-2020 _ 18

Graphique 9 Évolution de la population par pays (en millions) ...................................................................19

Graphique 10 Taux de fécondité des pays ouest-africains les plus avancés, 1960-2020 _................... 20

Graphique 11 Taux de fécondité des pays ouest-africains les moins avancés, 1960-2020_................. 21

Graphique 12 Fertilité et urbanisation en Afrique du Nord et de l'Ouest, 2020 …............................. 22

Graphique 13 Taux d'urbanisation par pays, 2020 _ 23

Graphique 14 Taux d'urbanisation régional, 1950-2020 24

Graphique 15 Dynamiques de peuplement en Afrique de l'Ouest, 1950-2020 _ _........................... 24

Graphique 16 Croissance et volume de la population urbaine par région, 1950-2020_.......................... 25

Graphique 17 Croissance et volume de la population rurale par région, 1950-2020 _ _ _ . 26

Graphique 18 Croissance démographique comparée du Sahel et de l'Afrique du Nord, 1950-2050 ...27

Graphique 19 Projection du nombre d'habitants par pays, 1950-2050 (en millions d'habitants) _...... 27 



\section{Introduction}

L'Afrique connaît une croissance démographique sans précédent dans l'histoire du continent. Si les taux de croissance se poursuivent, le continent comptera 2.5 milliards d'habitants en 2050, soit le double de la population actuelle (Nations Unies, 2019[1]). Cette croissance démographique se double d'une croissance urbaine spectaculaire. Alors que la population urbaine d'Afrique est passée de 27 millions à 567 millions de personnes entre 1950 et 2015, les villes absorberont deux tiers de la croissance démographique projetée d'ici à 2050 (OCDE/CSAO, 2020[2]).

Alimentée par une forte croissance naturelle et d'intenses migrations régionales, l'urbanisation conduit à des transformations qui dépassent la simple distribution géographique de la population et sa structure démographique. La croissance et la multiplication des villes sont aussi synonymes de changements importants pour l'organisation sociale des populations africaines, leurs activités économiques et leurs équilibres politiques. Comme dans le reste du monde, l'urbanisation permet un accès plus rapide aux services de santé et d'éducation, aux marchés et aux institutions qui favorisent les droits humains et la démocratie.

Dans ce contexte, cette note propose une analyse factuelle et rétrospective des relations entre urbanisation et démographie en Afrique du Nord et de l'Ouest. Elle actualise les travaux menés au sein du Club du Sahel et de l'Afrique de l'Ouest (CSAO) de l'OCDE en matière de peuplement humain (OCDE/CSAO, 2014[3] ; 2013[4]) et d'urbanisation (Moriconi-Ebrard, Harre et Heinrigs, 2016[5] ; OCDE/CSAO, 2020[2]) depuis une dizaine d'années. La note décrit tout d'abord le processus de transition démographique qui marque le passage d'un équilibre à forte natalité et forte mortalité à un nouvel équilibre marqué par des taux faibles. Sur la base d'une analyse à long terme des tendances régionales, elle discute ensuite dans quelle mesure les stades théoriques de la transition démographique s'appliquent aux pays d'Afrique du Nord et de l'Ouest. Enfin, la note illustre les relations entre urbanisation et fécondité et met en évidence certains grands changements démographiques projetés à l'horizon 2050. 


\section{La transition démographique}

L'évolution historique de la population mondiale est conditionnée par deux grands facteurs démographiques : le nombre de naissances et le nombre de décès. Jusqu'à la fin du XVIIIe siècle, tous les pays du monde ont connu une forte natalité combinée à une forte mortalité, caractéristiques d'un régime démographique traditionnel dans lequel les ménages compensaient le très grand nombre de décès par un nombre de naissances proches du maximum biologique. Durant cette période, l'équilibre précaire entre natalité et mortalité était sans cesse mis en péril par les conflits, les épidémies et les famines, avec pour conséquence une très faible augmentation de la population.

La révolution industrielle, l'urbanisation, les progrès sanitaires et les avancées médicales observés au cours des deux derniers siècles ont conduit à une chute rapide des taux de mortalité, suivie d'une chute exceptionnelle de la natalité. Ce passage d'un régime démographique traditionnel à un nouvel équilibre, marqué par une très faible mortalité et natalité, est connu sous le nom de transition démographique (Graphique 1). Trois grandes phases théoriques sont observées dans ce processus.

Graphique 1.

Phases théoriques de la transition démographique

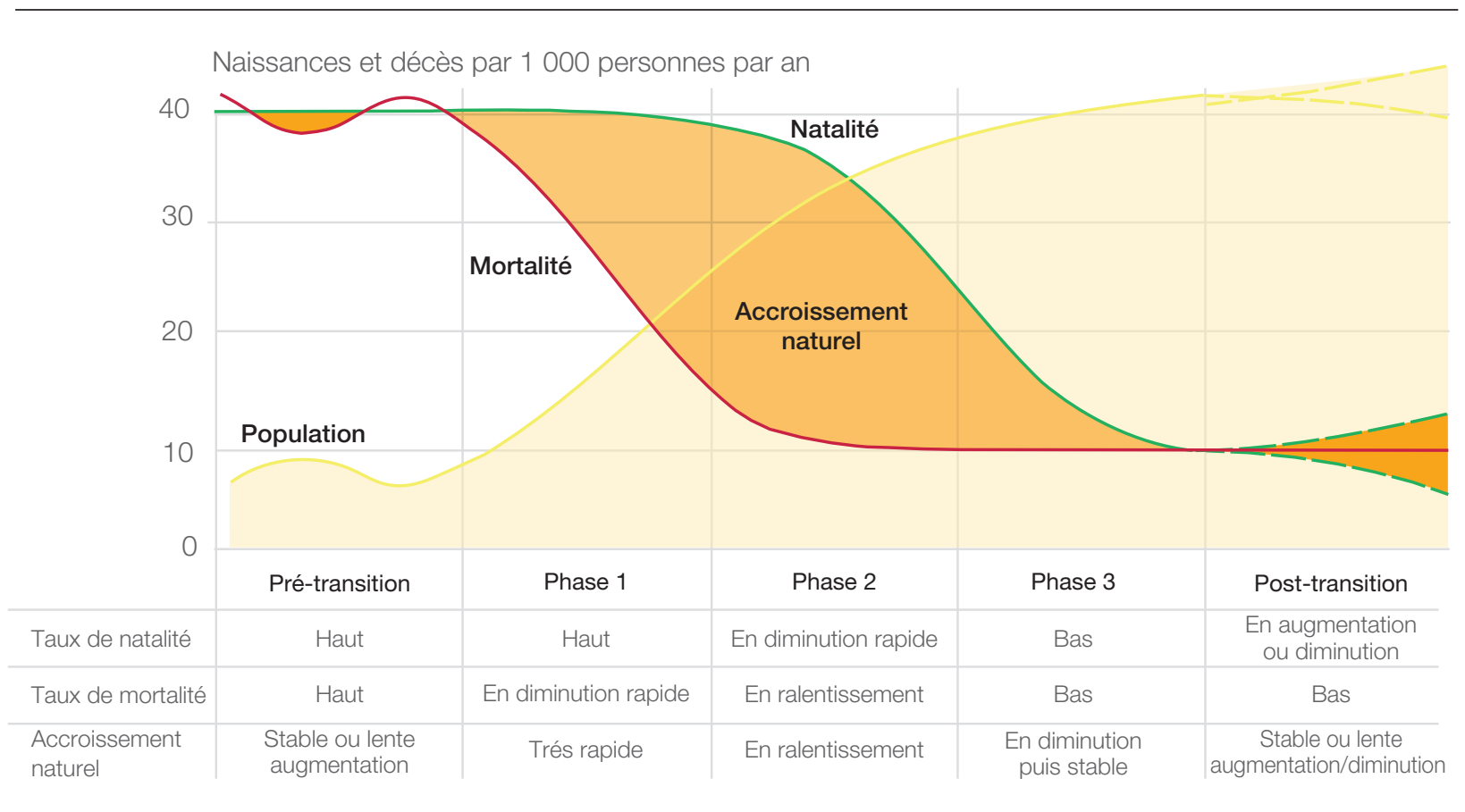

Source : Our World in Data (2021[6]) et Doignon (2020[7]), adapté par l'auteur. 
- Dans la première phase, la mortalité exprimée sous la forme du nombre de décès pour 1000 habitants par an connaît une baisse rapide, particulièrement la mortalité infantile. L'accès aux soins médicaux et une meilleure alimentation font notamment chuter le nombre de décès de 40 \% à $15 \%$. Durant cette première phase, la natalité exprimée sous la forme du nombre de naissances pour 1000 habitants par an reste très élevée, aux environs de 40 \%. Le décalage observé entre chute de la mortalité et chute de la natalité s'explique par l'inertie de nombreuses valeurs morales et l'évolution lente des modèles familiaux, qui valorisent encore les grandes familles et limitent l'émancipation féminine.

- Dans la seconde phase de la transition démographique, les changements sociaux et moraux, l'augmentation des emplois non agricoles et l'accès aux contraceptifs modernes encouragent une baisse rapide de la natalité, qui passe de 40 \% à $25 \%$. La mortalité continue de reculer, à un rythme plus lent cependant, et se situe désormais entre 10-15\%o. L'accroissement naturel de la population, qui avait atteint des sommets au cours de la première phase de la transition, ralentit également du fait de la chute rapide du nombre de naissances.

- La troisième phase de la transition démographique est marquée par une convergence graduelle entre taux de natalité et mortalité, qui atteignent environ 10 \%.. En conséquence, l'accroissement naturel de la population est faible durant cette phase qui conduit graduellement à un nouvel équilibre démographique.

Une fois la transition démographique achevée, natalité et mortalité atteignent des niveaux bas synonymes de stabilité démographique. Alors que l'évolution de la population en phase pré-transitionnelle était dictée par la mortalité, c'est la natalité qui détermine maintenant les changements démographiques. Dans un certain nombre de pays, l'aggravation des conditions économiques peut conduire à un déclin démographique si le recul de la natalité n'est pas compensé par l'émigration internationale. Dans d'autres pays, un changement dans les normes religieuses peut encourager certains ménages à avoir plus d'enfants, avec pour conséquence une légère croissance démographique. 


\section{Changements démographiques en Afrique du Nord et de l'Ouest}

Le processus de transition démographique connaît des variations nationales et régionales qui s'expliquent par le contexte social, politique et économique. La baisse de la mortalité, par exemple, est favorisée par l'urbanisation, qui encourage l'accès aux services de santé et d'éducation et par le passage d'une économie agricole à une économie plus orientée sur la production industrielle ou les services. Les politiques publiques peuvent aussi avoir un effet considérable sur la baisse de la natalité, de manière coercitive comme en Chine, ou par le biais d'incitations au planning familial comme au Bangladesh.

\section{UNE EXCEPTION DÉMOGRAPHIQUE ?}

Du fait de la diversité des situations nationales et régionales, il importe de connaitre dans quelle mesure les phases théoriques de la transition démographique s'appliquent aux pays nord et ouest-africains. L'analyse de l'évolution temporelle de la natalité et de la mortalité par pays de 1960 à 2020 montre que tous les pays de la région sont engagés dans la transition démographique, à des degrés divers. Aucun pays ne présente des signes de haute natalité et de haute mortalité caractéristiques du régime démographique traditionnel (pré-transition) en 2020. La transition démographique suit le modèle général observé dans le reste du monde, avec toutefois deux différences importantes en matière de natalité. En comparaison avec l'Asie du Sud-est et l'Amérique latine, la chute de la fécondité est plus tardive et plus lente en Afrique du Nord et de l'Ouest. Ces particularités, qui s'observent à l'échelle du continent, ont encouragé certains auteurs à évoquer une forme d'exception africaine au cours des années 2010 (Bongaarts, 2017[8]).

Les données les plus récentes incitent cependant à penser que même si elle n'est peut-être pas aussi rapide qu'ailleurs, la chute de la natalité se poursuit dans la décennie contemporaine et conduit à un nouvel équilibre démographique sur le continent à moyen terme (Lerch, 2019[9]). En d'autres termes, l'Afrique ne fait pas exception en comparaison internationale même si, dans certains pays, la natalité demeure supérieure à ce que le modèle 
théorique prédit pour chacune des phases. La lenteur relative de la chute de la fertilité en Afrique subsaharienne, particulièrement dans les zones rurales, conduit à une importante augmentation de la population totale, dont le nombre est régi par l'écart observé entre natalité et mortalité.

\section{DIVERSITÉ RÉGIONALE EN MATIÈRE DE TRANSITION DÉMOGRAPHIQUE}

L'analyse de l'évolution démographique met en évidence trois grands groupes de pays : (1) trois pays sahéliens parmi les plus pauvres et les moins urbanisés, (2) la majeure partie des autres pays sahéliens et d'Afrique de l'Ouest, (3) les pays nord-africains et le Cabo Verde.

- Avec des taux de natalité encore supérieurs à 40 \% et une mortalité en diminution rapide, le Niger, le Tchad et le Mali sont en première phase de transition démographique (Graphique 2). Cette situation est la plus favorable à l'accroissement naturel de la population nationale. Il n'est, dès lors, pas surprenant de constater que ces trois pays connaissent parmi les plus forts taux de croissance démographique du continent, soit 3.8 \% au Niger et $3.0 \%$ au Tchad et au Mali en 2019. Ces taux de croissance conduisent à un doublement de la population en moins de 19 ans au Niger et en moins de 24 ans au Tchad et au Mali. Avec 8-12 décès pour 1000 habitants, la mortalité dans ces trois pays demeure moins élevée que ce que le modèle théorique considère comme représentatif de la première phase de transition (15-40\%o). En revanche, le taux de natalité, compris entre 41-45 naissances pour 1000 habitants, est supérieur au modèle théorique (40\%). Ces taux n'ont rien d'inhabituel dans la région : les pays d'Afrique du Nord et le Cabo Verde ont eu des taux de natalité supérieurs à 40 \% jusqu'au début des années 80 (Graphique 4).

- Les autres pays du Sahel et d'Afrique de l'Ouest sont engagés dans la seconde phase de la transition démographique caractérisée par une diminution rapide de la natalité et un ralentissement de la chute de la mortalité. Seuls les taux observés au Nigéria et en Sierra Leone correspondent strictement au modèle théorique dans lequel la natalité atteint de 25-40 \%o et la mortalité de 10-15\%. Les autres pays connaissent une mortalité comprise entre 5-10 \%, plus faible que ce que le modèle théorique prédit durant cette phase (10-15\%), un signe qui annonce le prochain stade de la transition démographique. 
Graphique 2.

Phases de la transition démographique en Afrique du Nord et de l'Ouest

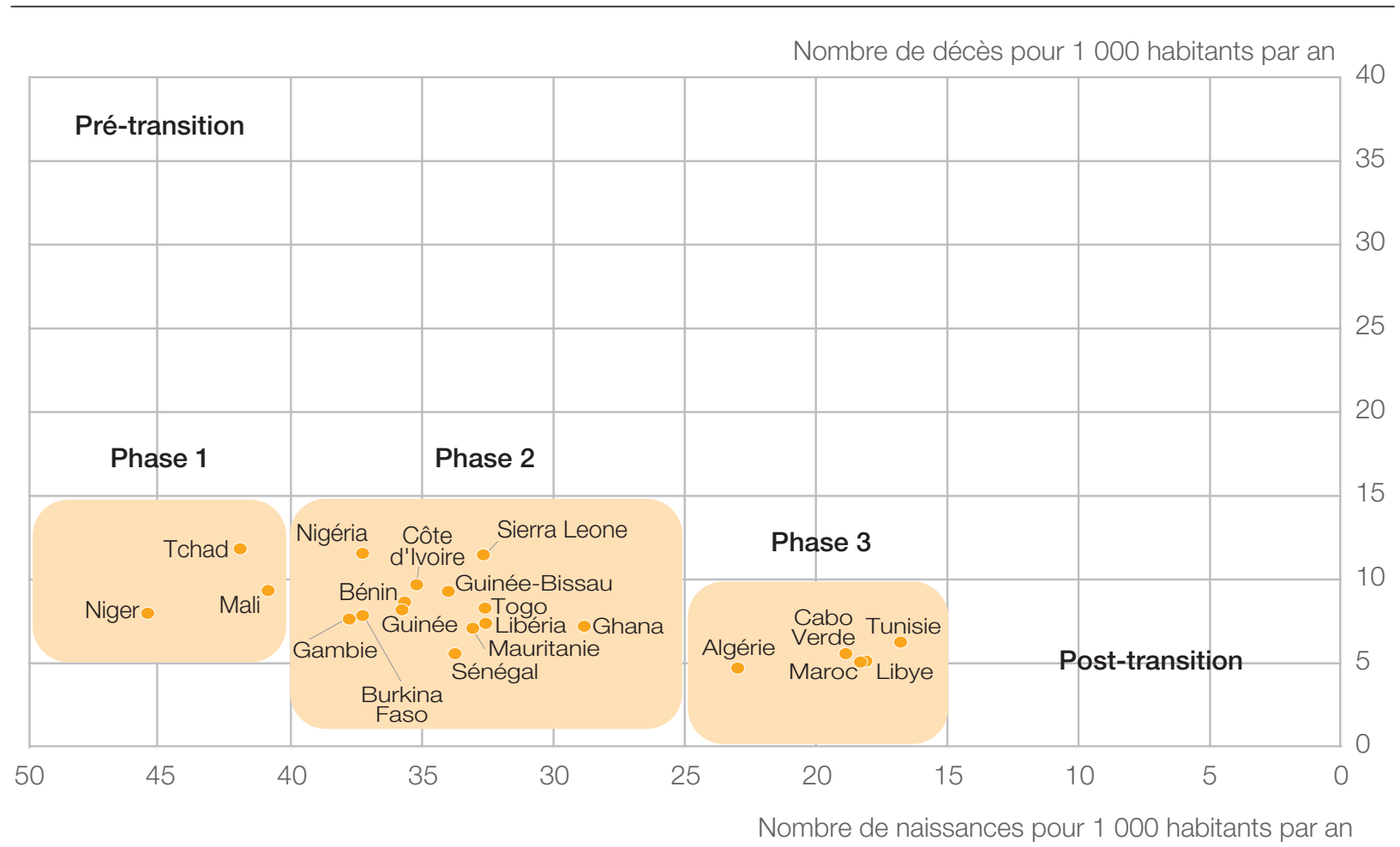

Source : Nations Unies (2019[1]). Calculs de l'auteur.

- Les pays d'Afrique du Nord et le Cabo Verde sont les plus avancés dans la transition démographique. Leurs taux de mortalité inférieurs à $10 \%$ correspondent globalement aux valeurs de la période post-transitionnelle du modèle théorique. Cependant, leur taux brut de natalité compris entre 17-23 \% est caractéristique de la troisième phase de la transition. Du fait du taux relativement élevé de natalité, la croissance naturelle de la population de ces pays est plus soutenue que dans les pays ayant atteint un nouvel équilibre démographique autour de 10 naissances et décès pour 1000 habitants, comme en Europe occidentale.

Des différences marquées sont observées dans la temporalité du processus de transition démographique entre les deux rives du Sahara (Graphique 3). Au Maroc, en Algérie, en Tunisie et en Libye, le déclin de la natalité, entamé à partir des années 60, est parmi les plus rapides du monde. Il a fallu près de deux siècles à la Suède pour atteindre un nouvel équilibre démographique (1750-1980), 60 ans à la Corée du Sud (1920-80) et seulement une cinquantaine d'années pour certains pays nord-africains comme la Tunisie (1950-2000). Dans les pays sahéliens, la natalité est stable ou en légère augmentation jusque dans les années 2000. Elle connaît une chute rapide depuis lors. La mortalité a connu une diminution rapide et continue dans les deux régions. Elle converge à des niveaux très bas, proches de $10 \%$ au Sahel et 5 \%o en Afrique du Nord. 
Graphique 3.

Transition démographique en Afrique du Nord et au Sahel, 1950-2020

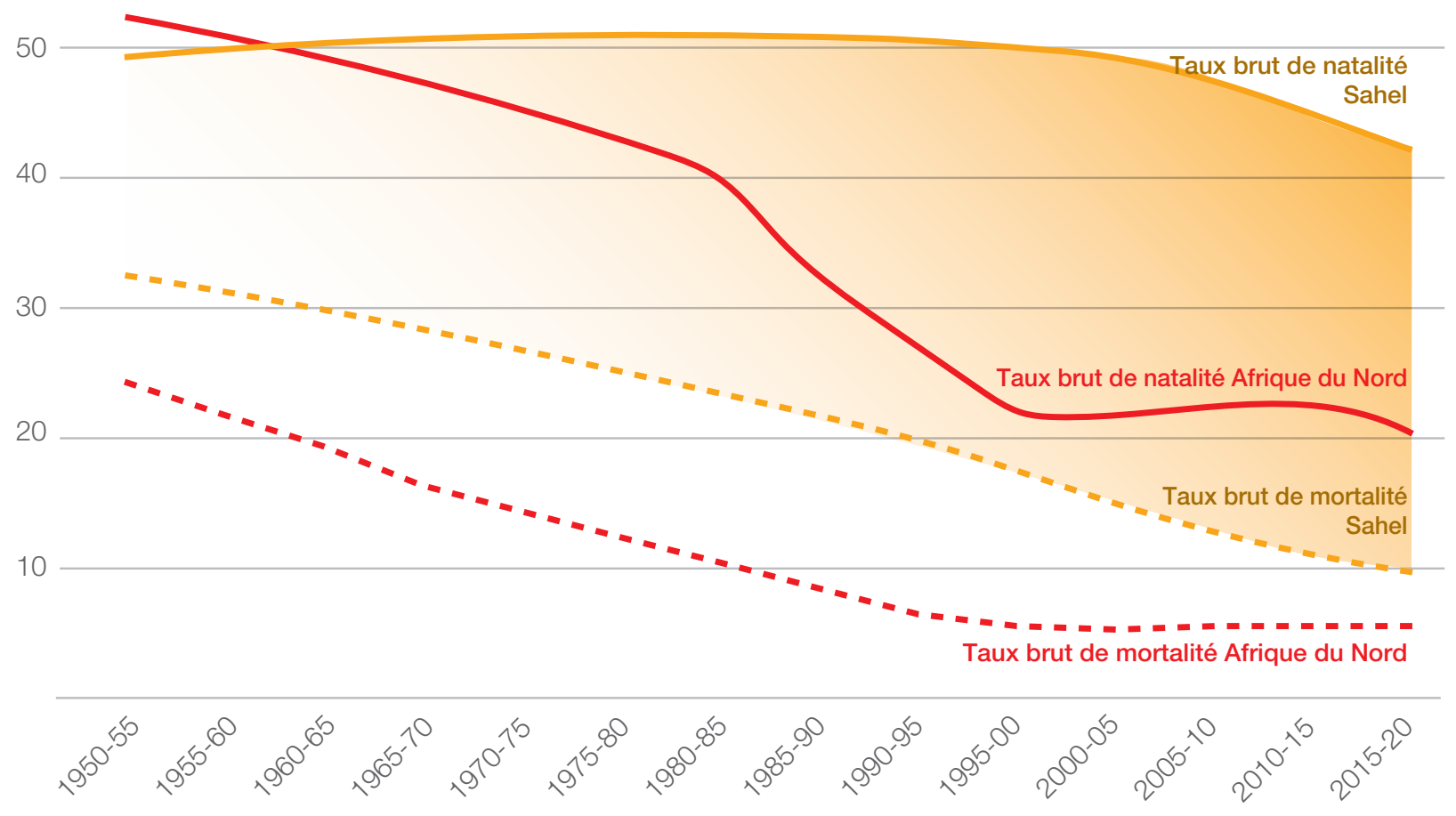

Source : OCDE/CSAO (2014[3]). Mis à jour par l'auteur à partir de Nations Unies (2019[1]).

\section{CONVERGENCE DES TAUX DE MORTALITÉ ET NATALITÉ EN AFRIQUE DU NORD ET DE L'OUEST}

La convergence historique de la mortalité et de la natalité est particulièrement évidente lorsque les valeurs annuelles de chaque taux sont présentées par pays au cours des 60 dernières années. Alors que leur taux de mortalité et de natalité étaient très différents en 1960, les pays d'Afrique du Nord et le Cabo Verde suivent une trajectoire convergente qui les amènent graduellement à un nouvel équilibre en 2020 (Graphique 4). La baisse de la natalité est moins linéaire et continue que celle de la mortalité. Une hausse du nombre de naissances pour 1000 habitants peut être observée au Cabo Verde entre 1975 et 1985, en Tunisie entre 2005 et 2014 ainsi que depuis le milieu des années 2000 en Algérie (Doignon, 2020[7]). 


\section{Graphique 4.}

Convergence de la natalité et mortalité en Afrique du Nord et Cabo Verde, 1960-2020

$60 \Longrightarrow$ Nombre de naissances et de décès pour 1000 habitants par an $\quad$ Algérie Cabo Verde
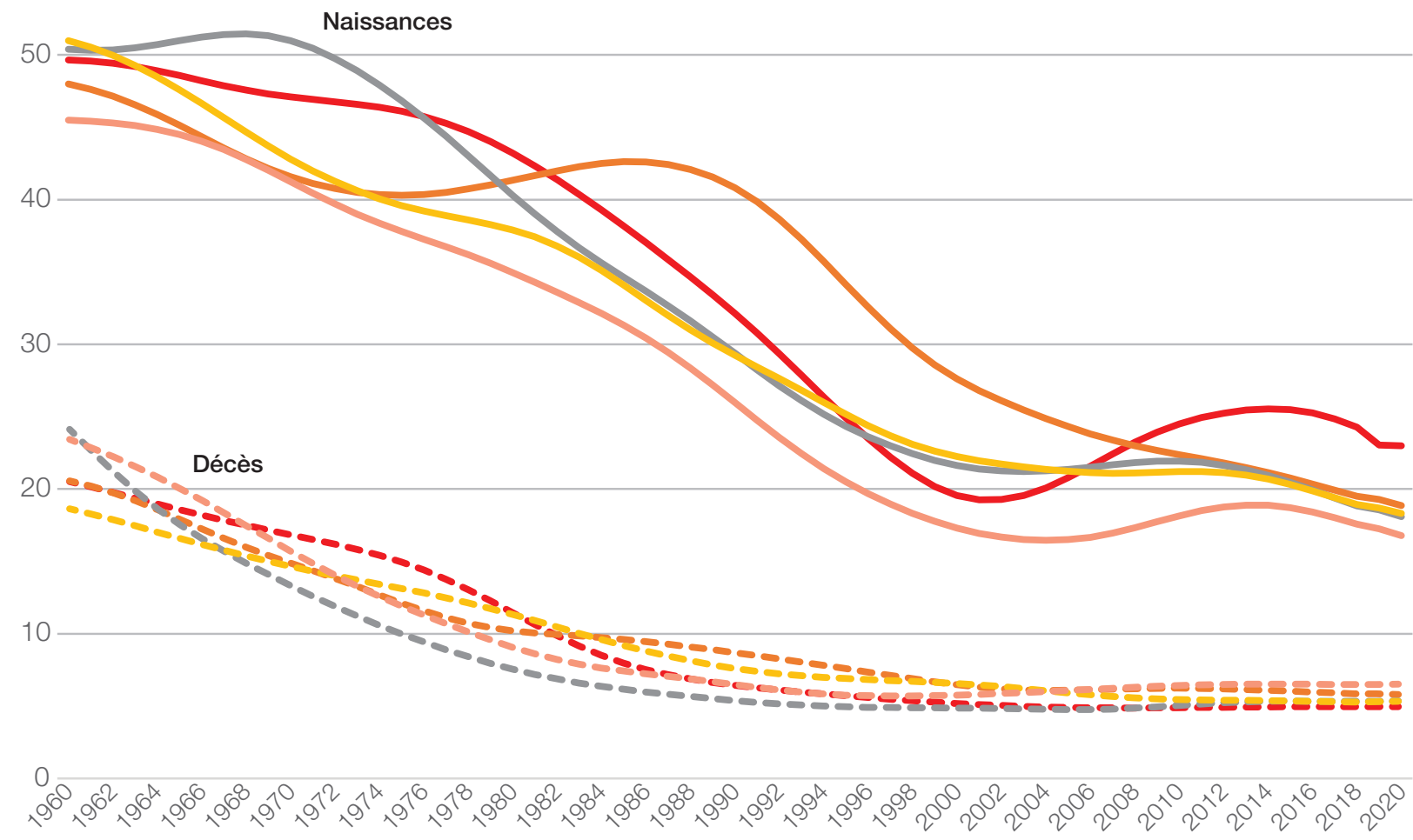

Source : Nations Unies (2019[1]). Calculs de l'auteur.

Les pays sahéliens convergent également du point de vue démographique (Graphique 5). La chute de la mortalité est rapide dans tous les pays. La natalité connaît également une baisse graduelle, sauf au Tchad, où une augmentation continue du nombre de naissances est observée jusqu'en 2000. Bien que le Niger ait débuté sa transition plus tardivement que les autres pays, ses progrès n'en sont pas moins rapides, particulièrement en termes de mortalité. Alors que le pays enregistrait plus de 26 décès pour 1000 habitants en 1980, ce taux est passé à 18 en 2000 et à 8 en 2020. Dans ce pays, la natalité est plus élevée qu'ailleurs mais connaît tout de même une baisse significative, passant de plus de 53 \% en 2000 à 46 \% en 2020. 
Graphique 5.

Convergence de la natalité et mortalité au Sahel, 1960-2020

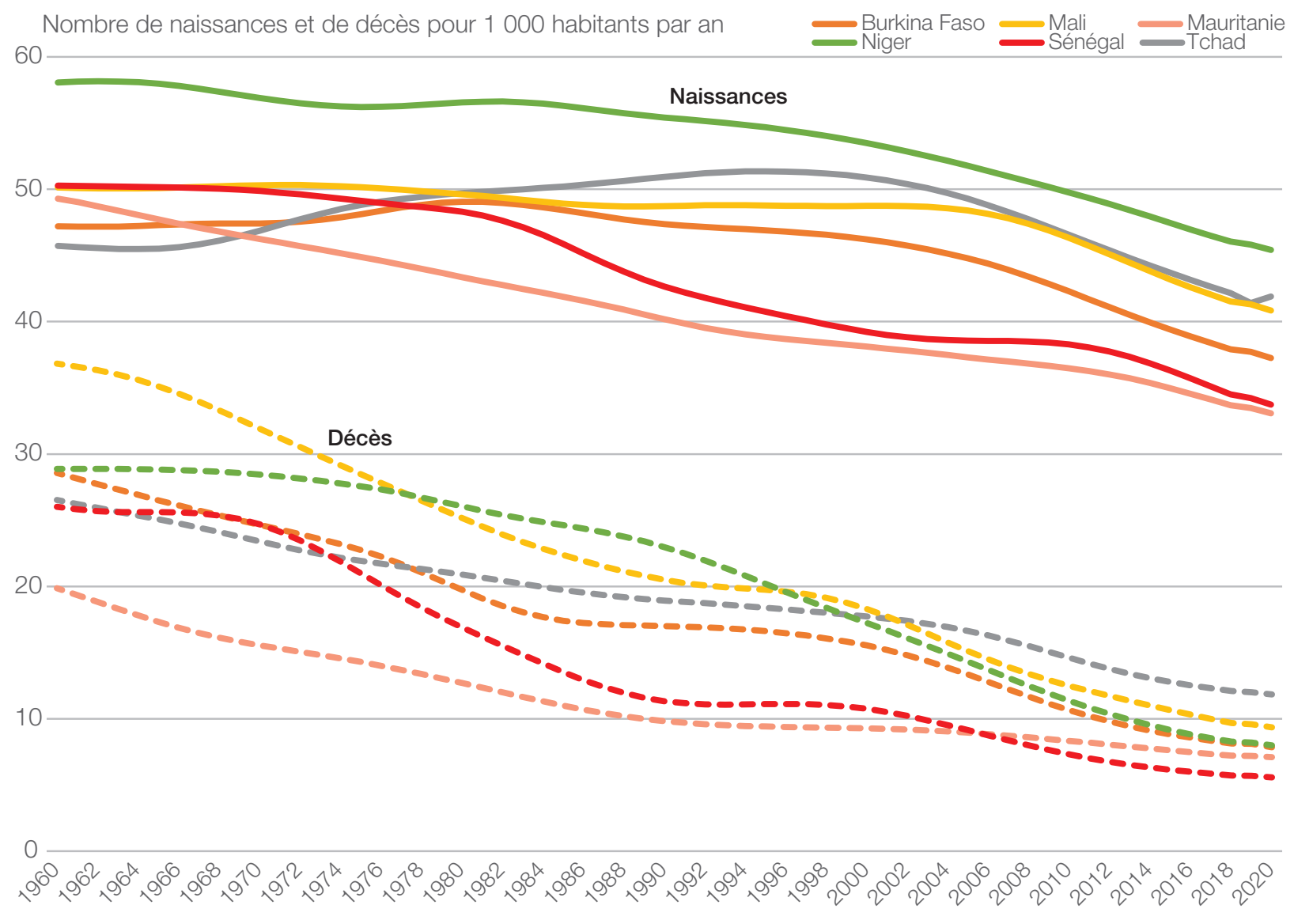

Sources : Nations Unies (2019[1]). Calculs de l'auteur.

\section{CROISSANCES DÉMOGRAPHIQUES NATIONALES}

En 2020, la population des huit pays saharo-sahéliens atteint 166 millions de personnes, contre 132 millions en 2010. L'Algérie, la Libye, le Maroc et la Tunisie rassemblent près de 100 millions d'habitants, soit une fois et demie plus que la population de leurs quatre voisins sahéliens, le Mali, la Mauritanie, le Niger et le Tchad (66 millions) (Graphique 6). 


\section{Graphique 6.}

Population par pays, 2020

Afrique du Nord (100 millions d'habitants)

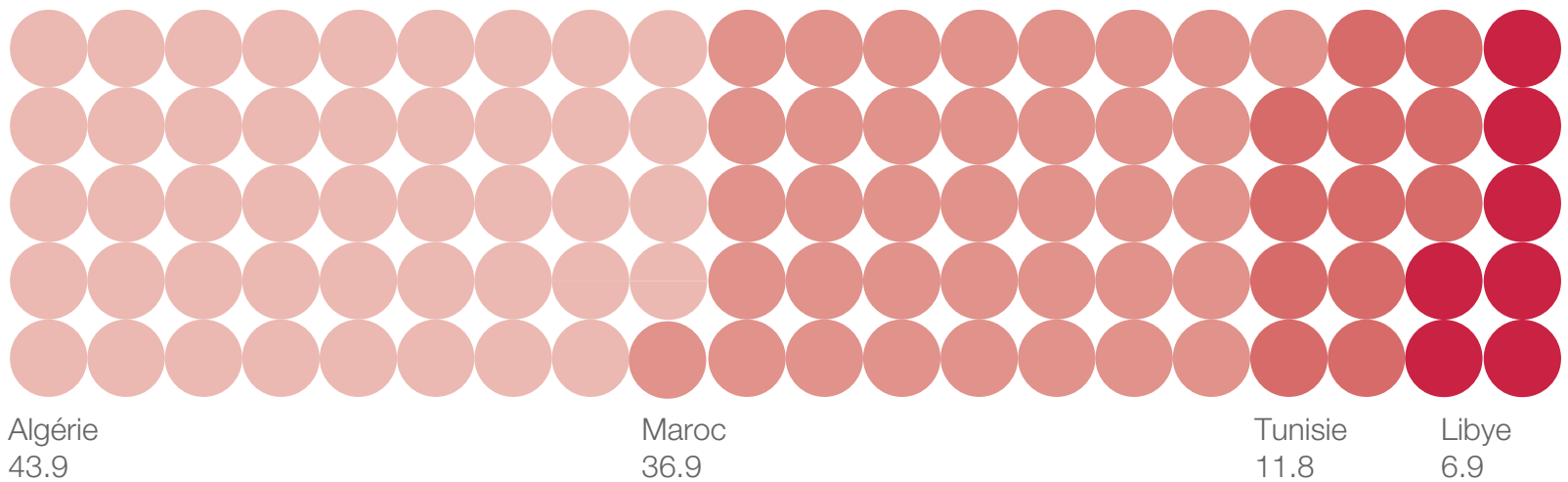

\section{Sahel (66 millions d'habitants)}

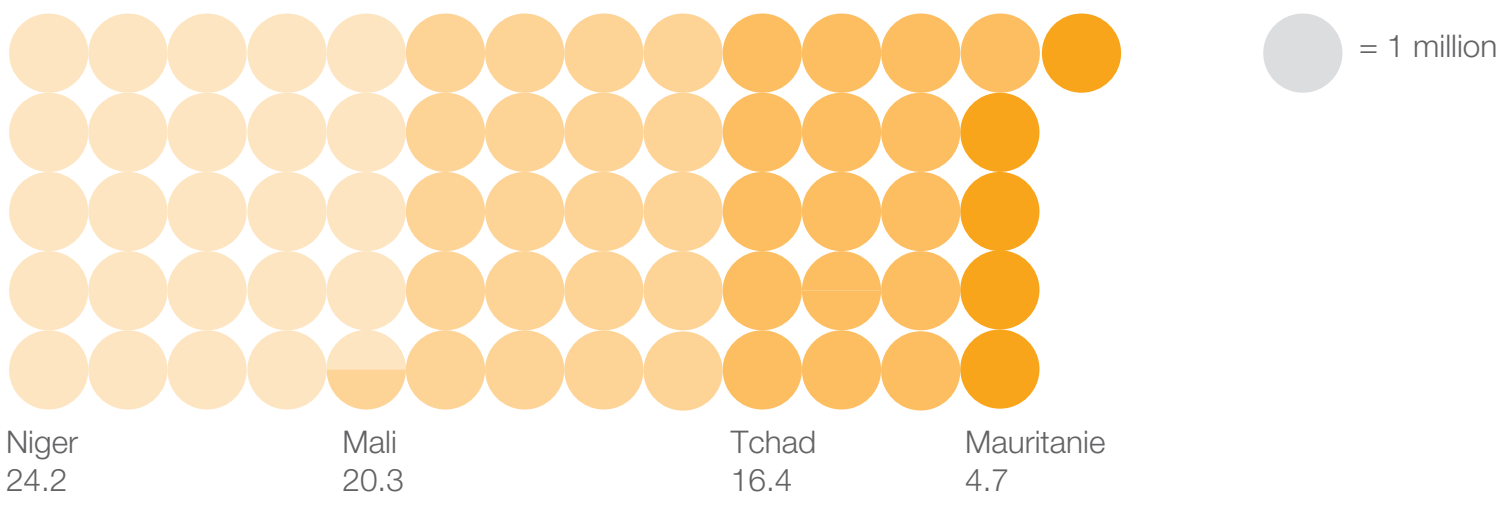

Source : OCDE/CSAO (2014[3]). Mis à jour par l'auteur à partir de Nations Unies (2019[1]).

Jusqu'au milieu des années 80, la croissance démographique des quatre pays nord-africains (Algérie, Libye, Maroc, Tunisie) est supérieure à celle enregistrée au sud du Sahara (Mali, Mauritanie, Niger et Tchad). Entre 1950 et 1980, la population nord-africaine augmente au taux annuel moyen de $2.7 \%$, passant de 29.0 millions d'habitants à 48.7 millions (Graphique 7). Dans la même période, la population du Sahel augmente au taux annuel moyen de $2.0 \%$, passant de 12.3 à 18.6 millions d'habitants. Les taux de croissance des deux régions divergent à partir des années 90. Entre 1990 et 2010, la population sahélienne croît au taux annuel moyen de $3.3 \%$, doublant quasiment, de 23.7 millions à 45.2 millions d'habitants. Le taux de croissance de l'Afrique du Nord descend à 1.4 \% entre 2005 et 2010 avant de remonter légèrement à $1.6 \%$ au cours de la dernière décennie. 
Graphique 7.

Croissance démographique et population en Afrique du Nord et au Sahel, 1950-2020

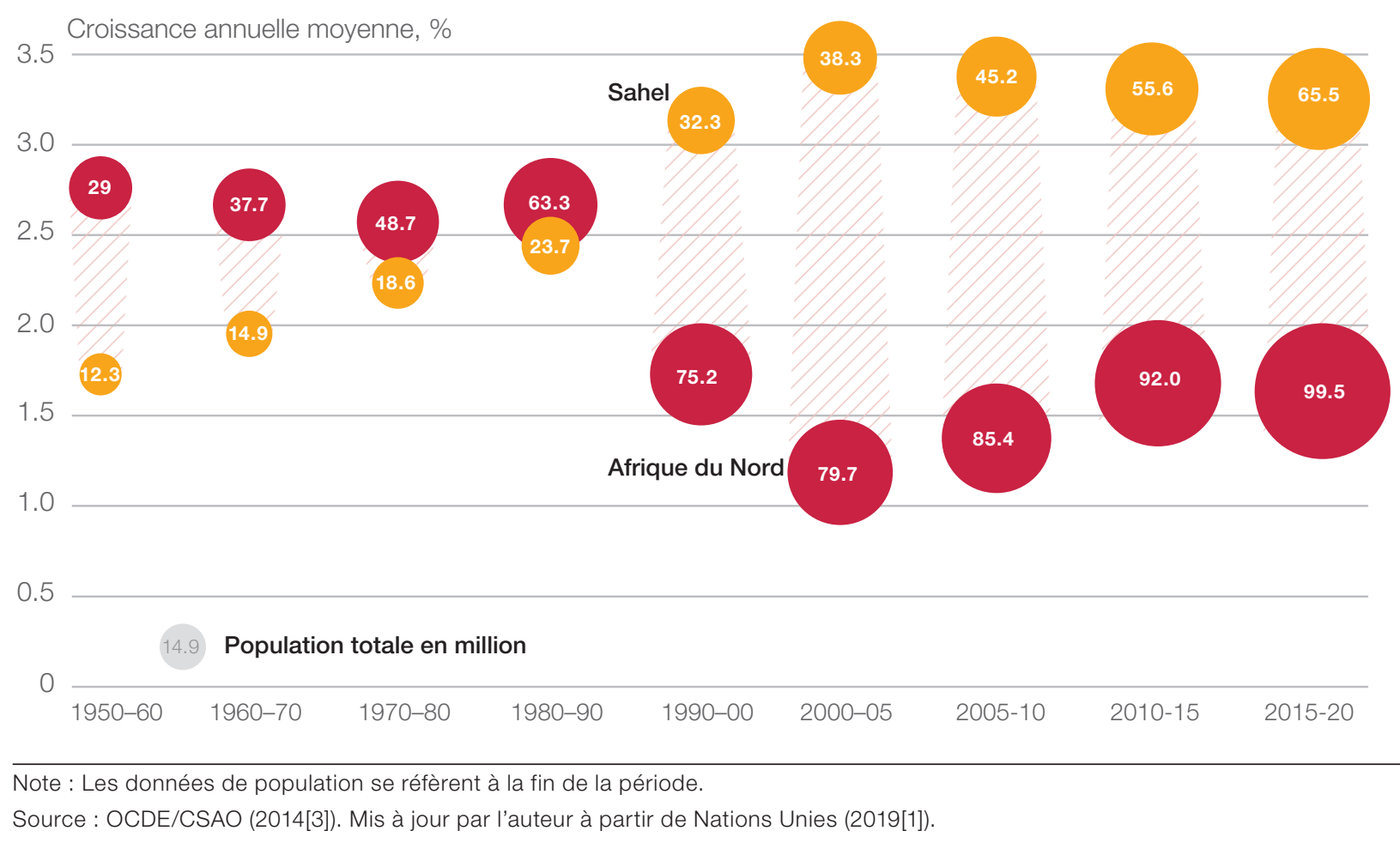

Les taux de croissance par pays confirment la divergence observée à l'échelon régional entre Afrique du Nord et Sahel (Graphique 8). Alors que les pays situés au nord du Sahara sont en phase finale de la transition démographique, ceux du Sahel sont engagés dans la première ou seconde phase synonyme de forte croissance. Entre les années 50 et 80, les taux de croissance annuels moyens les plus forts sont tout d'abord enregistrés en Libye et en Algérie, puis en Mauritanie et au Niger. À partir de 1990, aucun pays d'Afrique du Nord n'enregistre de taux annuel moyen supérieur à $3.0 \%$. Bien au contraire, l'évolution démographique de cette région est marquée par un recul très important de la croissance, qui atteint $1.1 \%$ par an en Tunisie, $1.3 \%$ au Maroc, $1.4 \%$ en Libye et $2.0 \%$ en Algérie. Le Sahel connait une évolution opposée : le rythme de la croissance démographique accélère à partir des années 90 dans tous les pays, jusqu'à atteindre un maximum de 4.0 \% par an au Niger entre 2010 et 2015, la valeur la plus forte enregistrée dans l'histoire moderne du Sahel. Les cinq dernières années sont marquées par un recul de la croissance démographique, qui atteint aujourd'hui 2.8 \% par an en Mauritanie, $3.0 \%$ au Mali, $3.3 \%$ au Tchad et $3.9 \%$ au Niger. 
Graphique 8.

Taux de croissance démographique par pays, 1950-2020

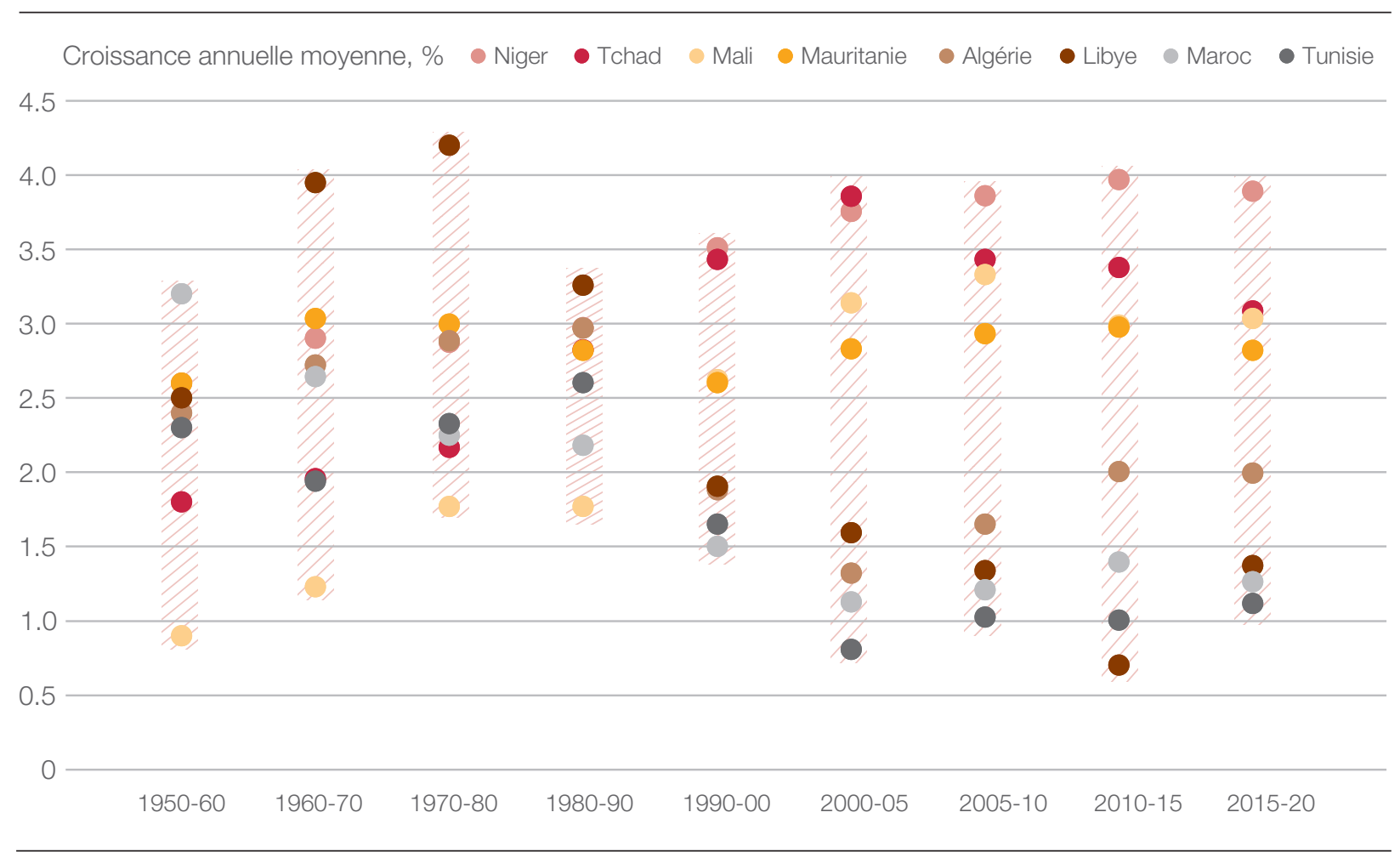

Note : Les données de population se réfèrent à la fin de la période.

Source : OCDE/CSAO (2014[3]). Mis à jour par l'auteur à partir de Nations Unies (2019[1]).

Avec près de 44 millions d'habitants en 2020, l'Algérie est le pays le plus peuplé de la région (Graphique 9). Il devance le Maroc, qui compte presque 37 millions d'habitants en 2020, depuis 1990. La vitalité de la population algérienne s'explique par un regain de la fertilité observé depuis le milieu des années 2000, qui a fait passer le nombre d'enfants par femme de 2.4 en 2005 à 3.1 en 2020. Au Maroc, au contraire, la fertilité moyenne est en baisse continue depuis les années 60. La croissance exceptionnelle de la population nigérienne explique que le pays soit le troisième plus peuplé de la région à partir des années 90, avec plus de 24 millions d’habitants en 2020, contre moins de 6 en 1980. Au cours des 20 dernières années, le Tchad et le Mali ont tous deux doublé leur population nationale, dépassant 16 et 20 millions en 2020. Avec 12 millions d'habitants, la Tunisie est presque deux fois plus peuplée que la Libye (7 millions). La Mauritanie compte moins de 5 millions d'habitants et demeure le pays le moins peuplé de la région. 
Graphique 9.

Évolution de la population par pays (en millions)

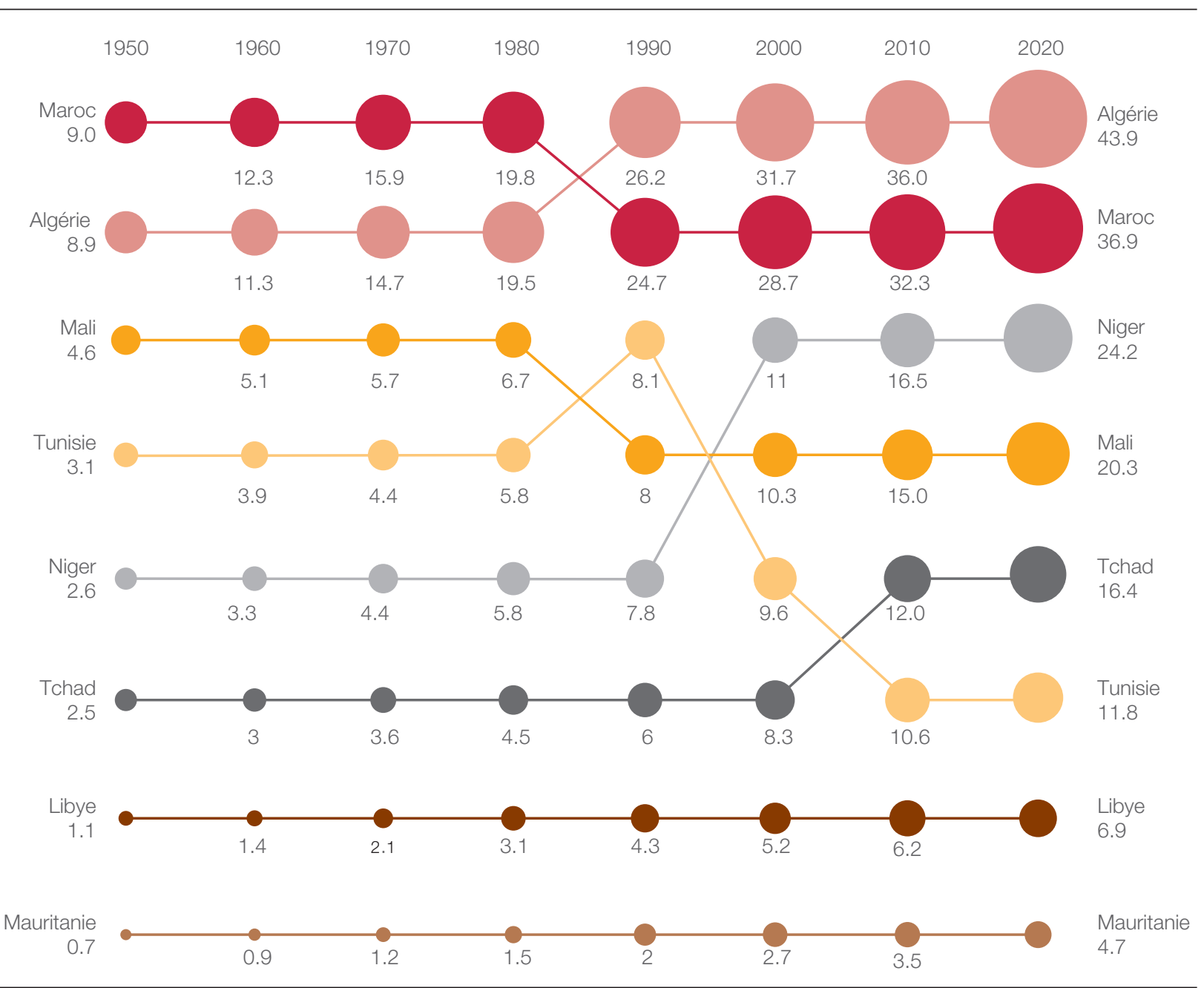

Source : OCDE/CSAO (2014[3]). Mis à jour par l'auteur à partir de Nations Unies (2019[1]). 


\section{UNE BAISSE DE LA FÉCONDITÉ GÉNÉRALISÉE EN AFRIQUE DE L'OUEST}

Les taux de fécondité ont chuté dans tous les pays ouest-africains au cours des dernières décennies. À l'échelle régionale, le nombre d'enfants par femme est passé de 6.6 en 1960 à 6.0 en 2000 et à 4.7 en 2020. Trois grands groupes de pays peuvent être identifiés selon leur trajectoire démographique.

- Dans les pays les plus avancés dans la transition démographique comme le Cabo Verde, la Côte d'Ivoire, le Ghana, le Libéria, la Mauritanie, le Togo et le Sénégal, la chute de la fécondité est antérieure au milieu des années 80 (Graphique 10). Le nombre d'enfants par femme y atteint 4.5 en 2020, à l'exception du Cabo Verde, dont le profil démographique se rapproche des pays nord-africains avec légèrement plus de deux enfants par femme.

Graphique 10.

Taux de fécondité des pays ouest-africains les plus avancés, 1960-2020

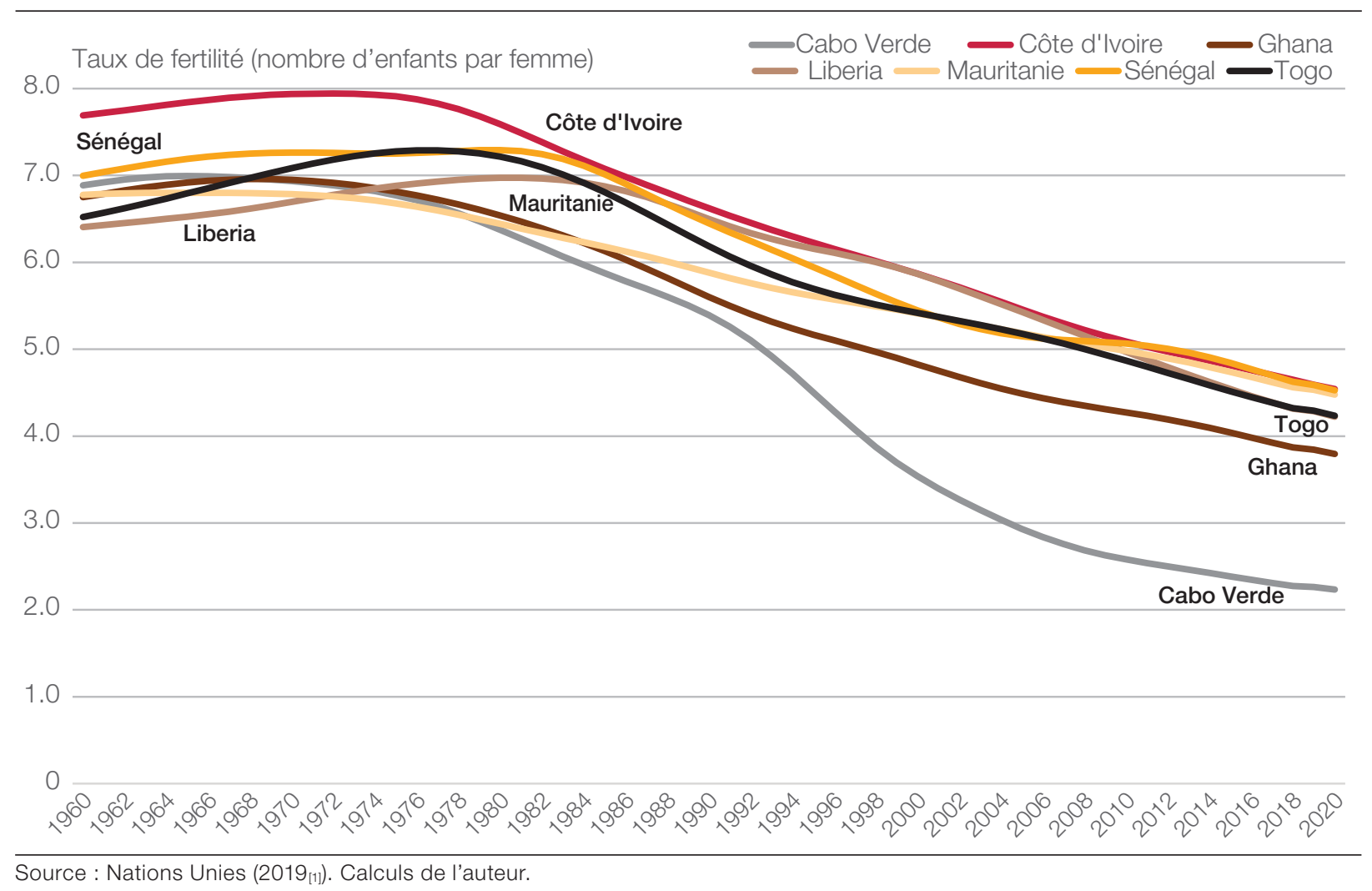


- Un second groupe de pays amorce sa chute de la fertilité à partir du début des années 90 (Bénin, Burkina Faso, Gambie, Guinée, GuinéeBissau, Nigéria, Sierra Leone). En 30 ans, le nombre d'enfants par femme y passe de 6.5 à 4.5 (Graphique 11).

- Trois pays se distinguent par une chute de la fécondité plus tardive, qui s'annonce à partir des années 2000 et s'accélère au cours des dernières années (Niger, Mali, Tchad). La progression la plus spectaculaire est observée au Tchad, où le nombre d'enfants par femme passe de 7.4 à 5.6 entre 2000 et 2020 (Graphique 11). Le Niger compte toujours la fécondité la plus élevée de la région, avec 6.8 enfants par femme en 2020, en recul toutefois par rapport à 2000 (7.7) et à 2010 (7.5).

Graphique 11.

Taux de fécondité des pays ouest-africains les moins avancés, 1960-2020

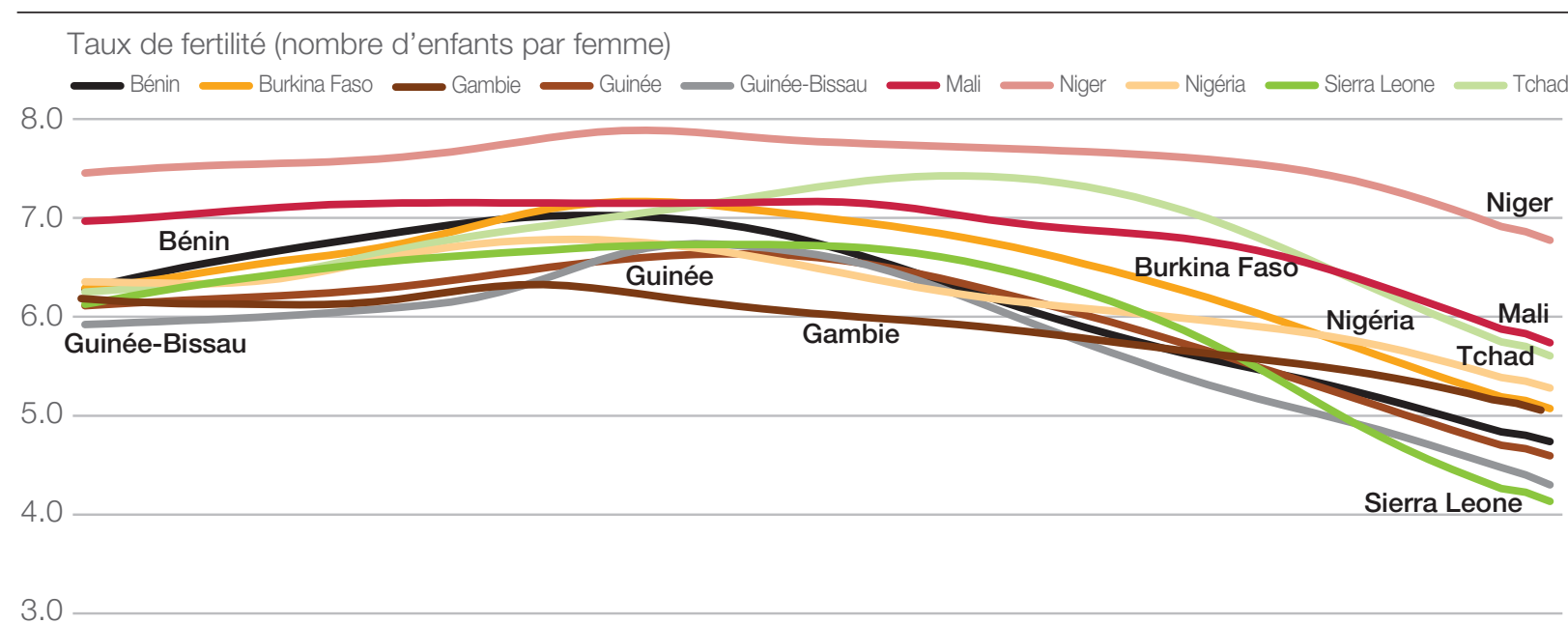

2.0

1.0

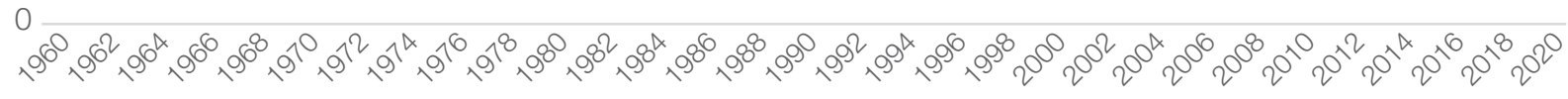

Source : Nations Unies (2019[1]). Calculs de l'auteur. 


\section{Démographie et urbanisation}

L'urbanisation est l'un des plus importants facteurs explicatifs de la chute de la mortalité et de la natalité observée dans le monde (Flückiger et Ludwig, 2017[10] ; Gries et Grundmann, 2018[11]). Les ménages urbains vivent plus longtemps et font moins d'enfants que les ménages ruraux. Bien que l'urbanisation favorise le processus de transition démographique, des différences significatives sont observées dans l'évolution de la population des villes selon leur taille en Afrique subsaharienne. Plus les villes sont grandes et moins la mortalité est élevée. De même, les ménages des grandes villes ont tendance à avoir des familles plus restreintes que ceux des petites villes (Corker, 2017[12]). Ces différentiels sont particulièrement importants en Afrique subsaharienne où l'immense majorité des villes (97 \%) comptent moins de 300000 habitants (OCDE/CSAO, 2020[2]).

\section{TRANSITION DÉMOGRAPHIQUE ET URBANISATION VONT DE PAIR}

En Afrique du Nord et de l'Ouest, les pays les plus urbanisés sont en règle générale ceux où le nombre d'enfants par femme est le moins élevé, comme en Libye, en Algérie, en Tunisie, au Cabo Verde et au Maroc (Graphique 12). À l'inverse, les pays les moins urbanisés connaissent encore une fertilité très importante, comme au Niger, au Tchad et au Burkina Faso. Le lien statistique entre fertilité et urbanisation est important : près de 70 \% de la variation de l'une des variables est expliqué par l'autre $\left(\mathrm{R}^{2}=0.7\right)$.

\section{Graphique 12.}

Fertilité et urbanisation en Afrique du Nord et de l'Ouest, 2020

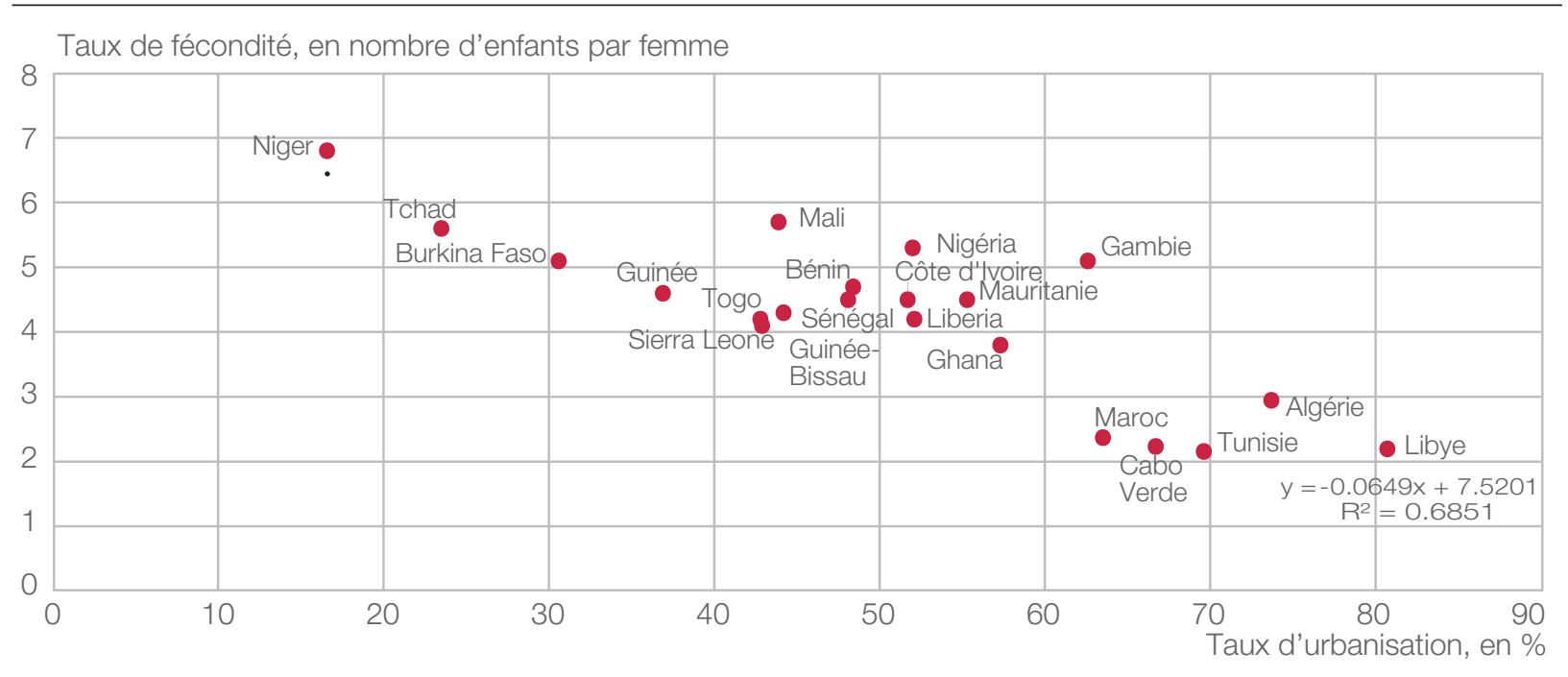

Source : Nations Unies (2019[1]). Calculs de l'auteur. 


\section{ESSOR DE L'URBANISATION}

La croissance urbaine a profondément modifié la distribution spatiale des populations d'Afrique du Nord et de l'Ouest au cours des dernières décennies. Le taux d'urbanisation est de $70 \%$ en Afrique du Nord (y compris l'Égypte) et de 47 \% en Afrique de l'Ouest en 2020. En Algérie et en Libye et Tunisie, près des trois quarts de la population vit en milieu urbain, tandis que moins de $30 \%$ de la population du Tchad et du Niger est urbaine (Graphique 13).

\section{Graphique 13.}

Taux d'urbanisation par pays, 2020

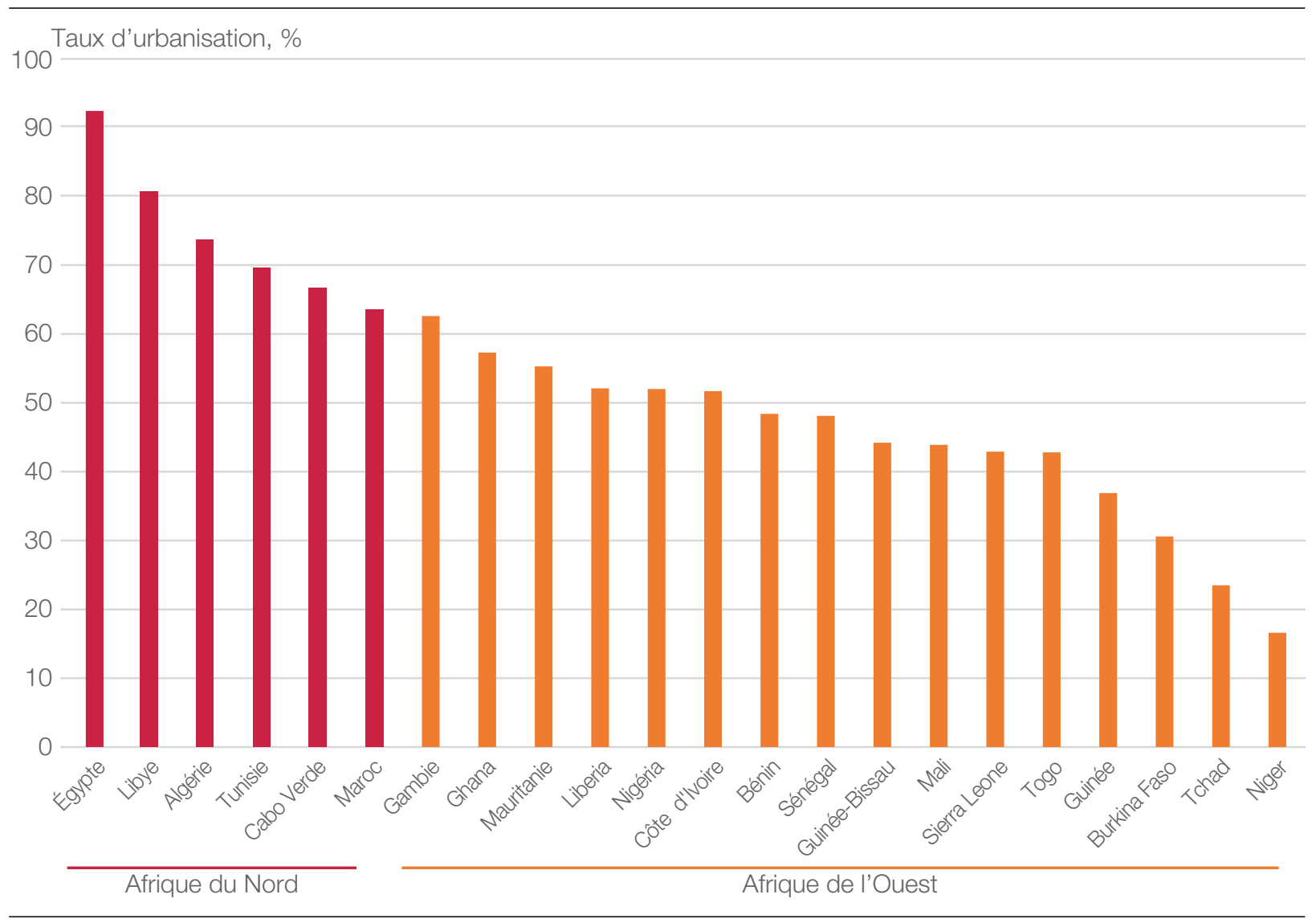

Sources : Banque mondiale (2021[13]), OCDE/CSAO (2020[2]). Calculs de l'auteur. 
Le taux d'urbanisation de l'Afrique du Nord est supérieur de $20 \%$ à celui de l'Afrique de l'Ouest (Graphique 14). Depuis 1950, l'écart entre les deux grandes régions est resté relativement constant.

Graphique 14.

Taux d'urbanisation régional, 1950-2020

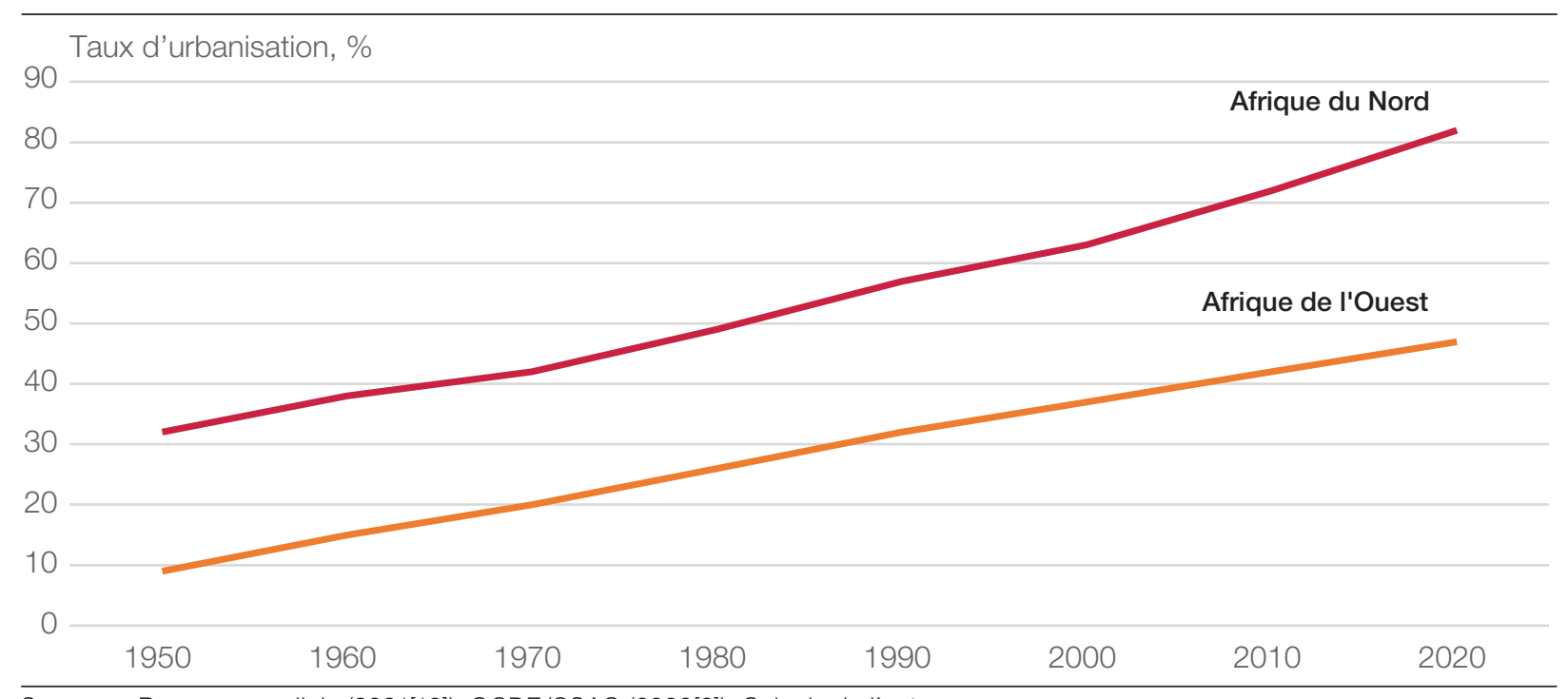

Sources : Banque mondiale (2021[13]), OCDE/CSAO (2020[2]). Calculs de l'auteur.

Au cours des 70 dernières années, l'augmentation de la population urbaine a été plus rapide que celle de la population rurale (Graphique 15). En 2020, le nombre de citadins approche les 200 millions dans la région alors que la population rurale dépasse les 220 millions.

Graphique 15.

Dynamiques de peuplement en Afrique de l'Ouest, 1950-2020

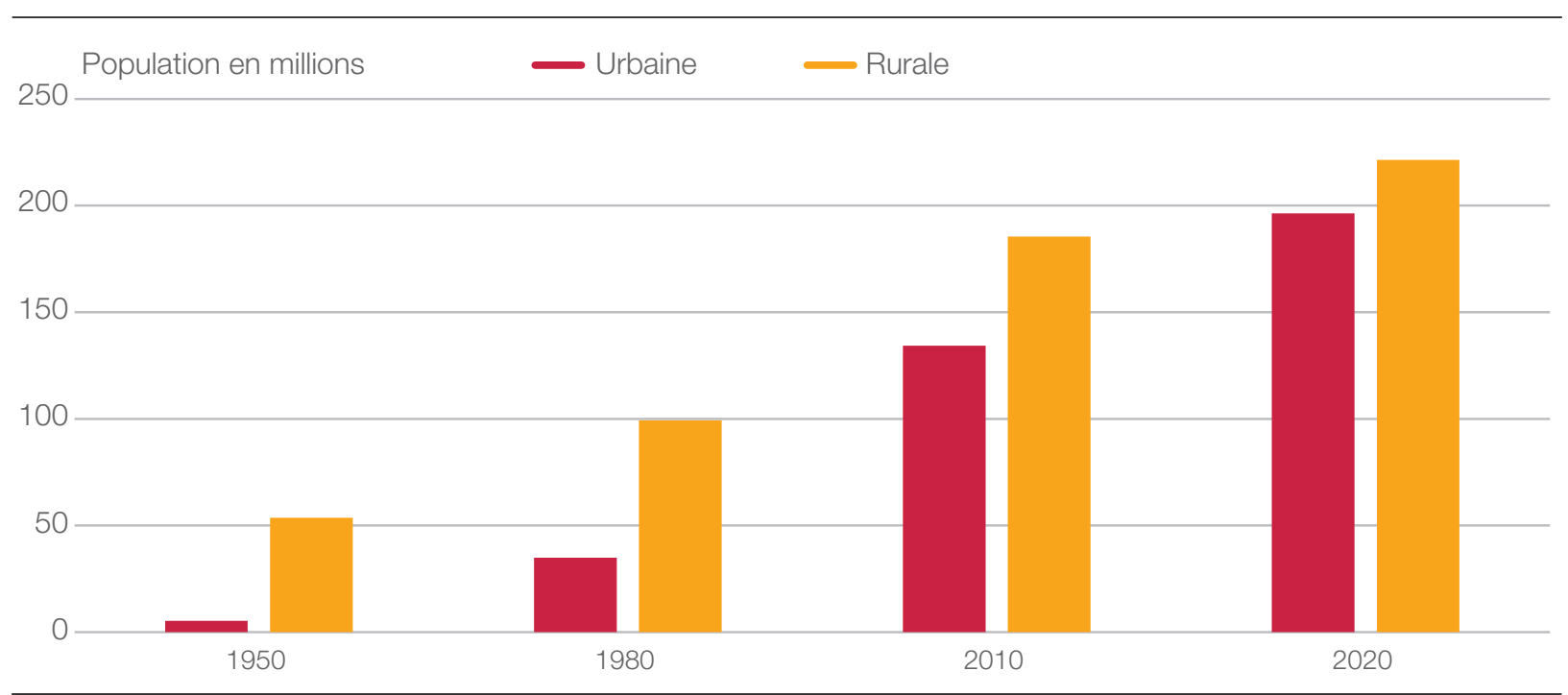

Note : Le Cabo Verde n'est pas inclus dans l'analyse.

Source : OCDE/CSAO (2014[3]). Mis à jour par l'auteur à partir de Banque mondiale (2021[13]). 
Au nord du Sahara, la population urbaine de l'Algérie, du Maroc, de la Libye et de la Tunisie compte presque 70 millions de personnes. La croissance urbaine de ces pays d'Afrique du Nord (+2.3\%) au cours de la décennie est de moitié inférieure à celle du Sahel (Graphique 16). Au sud du Sahara, la population urbaine du Mali, de la Mauritanie, du Niger, du Tchad est estimée à plus de 19 millions de personnes en 2020, en forte augmentation annuelle moyenne depuis 2010 (+5.2\%).

Graphique 16.

Croissance et volume de la population urbaine par région, 1950-2020

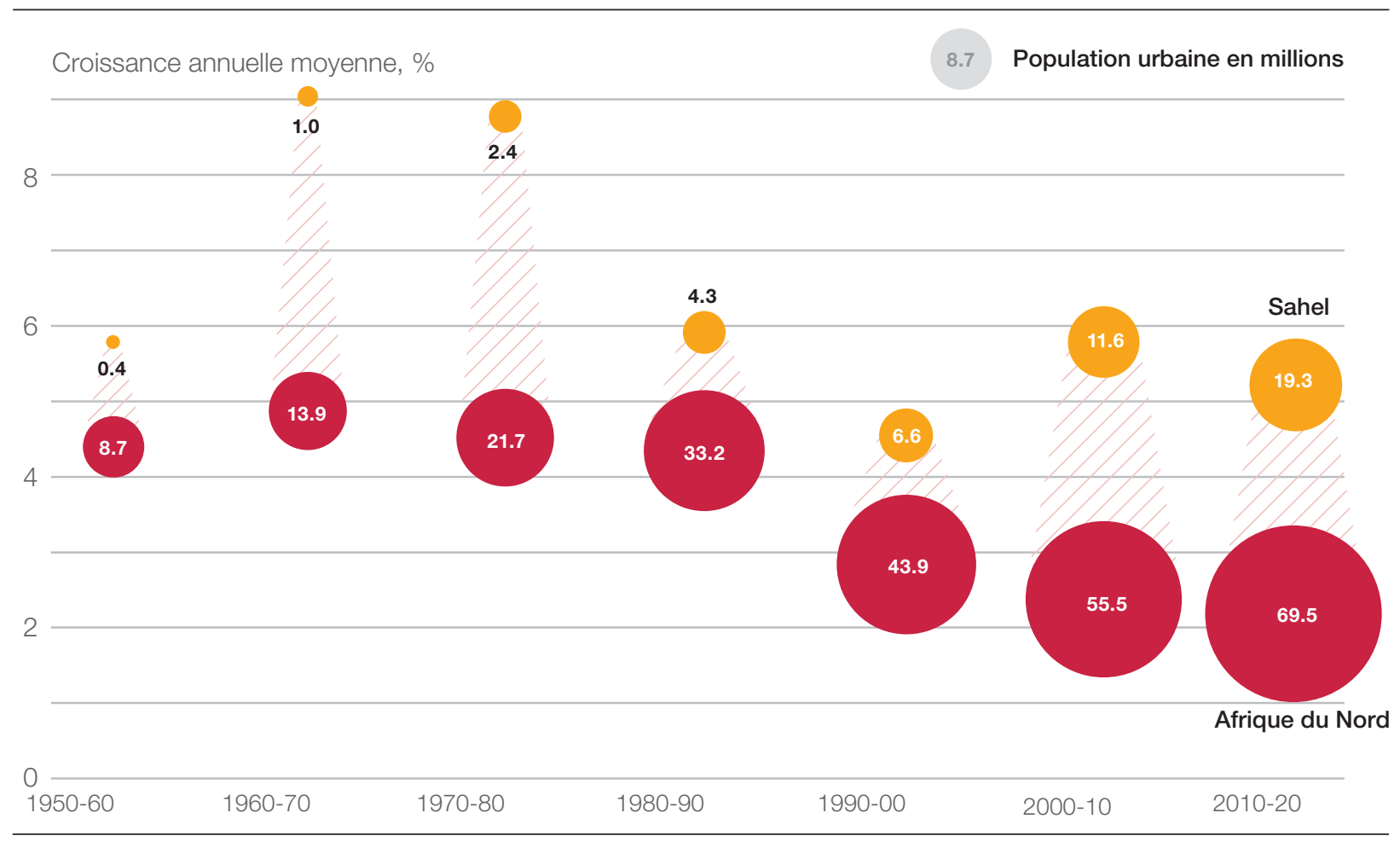

Note : Les taux de croissance annuelle moyens se réfèrent à la fin de la période d'observation.

Source : OCDE/CSAO (2014[3]). Mis à jour par l'auteur à partir de Nations Unies (2019[1]).

L'urbanisation rapide que connaît le Sahel s'accompagne d'une croissance continue des populations rurales, qui comptent plus de 46 millions d'habitants en 2020, contre 26 millions 20 ans plus tôt (Graphique 17). Le rythme de cette croissance rurale approche les $3 \%$ annuels. Dans les quatre pays d'Afrique du Nord, au contraire, l'essentiel de la croissance démographique est observé dans les villes. La croissance annuelle moyenne des populations rurales y est en diminution rapide depuis la fin des années 90. Elle fut négative entre 2000 et 2010 et nulle entre 2010 et 2020. Le nombre absolu de ruraux en Algérie, Maroc, Libye et Tunisie stagne aux environs de 30 millions depuis les années 2000. 
Graphique 17.

Croissance et volume de la population rurale par région, 1950-2020

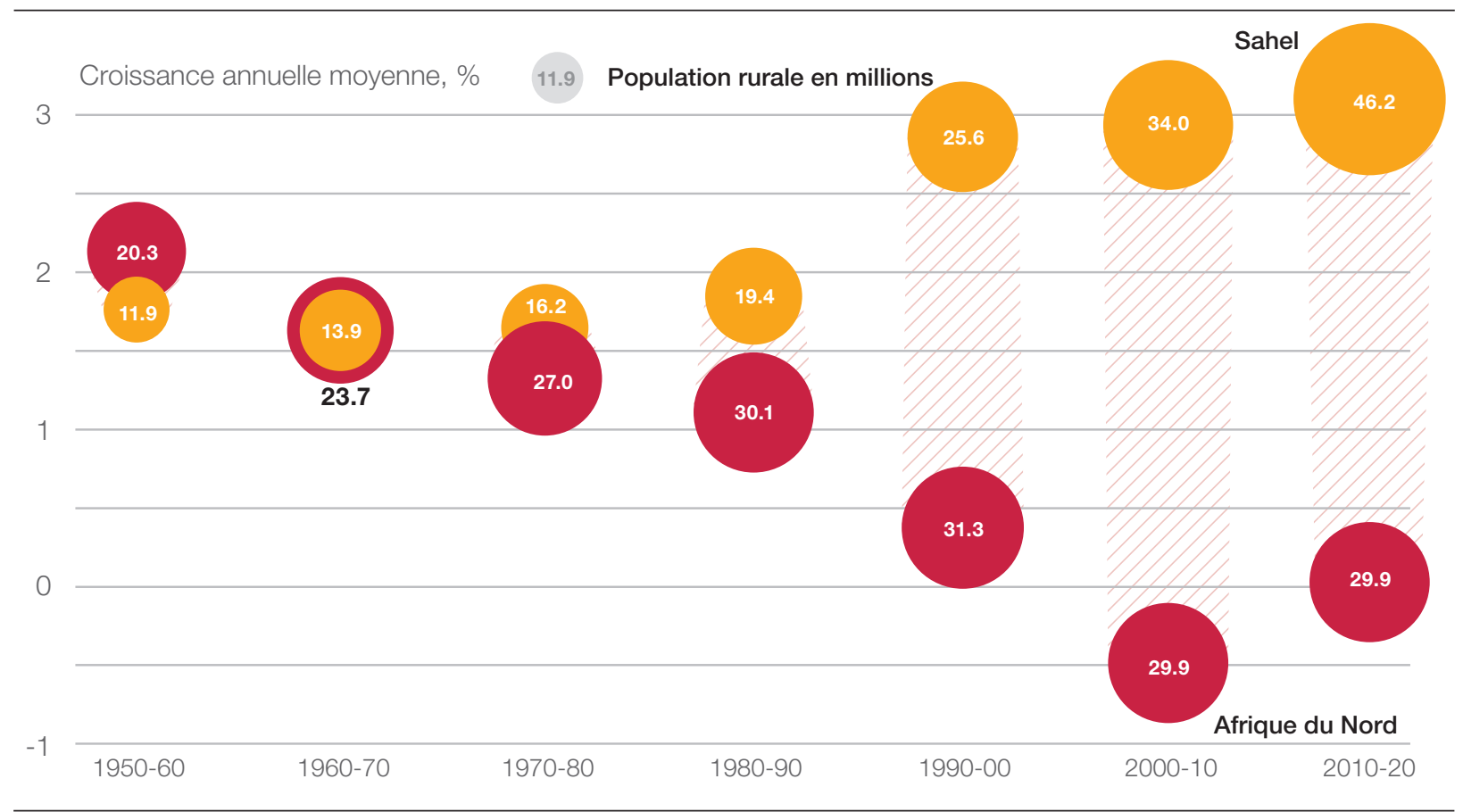

Note : Les taux de croissance annuelle moyens se réfèrent à la fin de la période d'observation.

Source : OCDE/CSAO (2014[3]). Mis à jour par l'auteur à partir de Nations Unies (2019[1]).

\section{CHANGEMENTS À L'HORIZON 2050}

D'ici à 2050, la population des quatre pays du Sahel devrait atteindre 141 millions de personnes, soit environ 10 millions de plus que celle des quatre pays situés au nord du Sahara (Graphique 18). La croissance annuelle moyenne devrait être légèrement supérieure à $2 \%$ par an au Sahel et inférieure à $1 \%$ par an en Afrique du Nord dans trente ans. Dans les deux régions, cette croissance devrait être inférieure à celle observée jusqu'en 2020 du fait du recul de la natalité lié au processus de transition démographique. L'écart entre taux de croissance entre pays sahéliens et nord-africains se maintiendra néanmoins.

La forte croissance démographique observée au sud du Sahara devrait conduire à une certaine redistribution du poids des pays dans la région d'ici à 2050 (Graphique 19). Avec 61 millions d'habitants, le Niger deviendra le pays le plus peuplé, dépassant très légèrement l'Algérie. Le Maroc sera le troisième pays le plus fortement peuplé d'Afrique du Nord et de l'Ouest, avec plus de 46 millions d'habitants en 2050. Le Mali et le Tchad, respectivement peuplés de 40 et 31 millions d'habitants, dépasseront très largement la Tunisie avec moins de 14 millions. 
Graphique 18.

Croissance démographique comparée du Sahel et de l'Afrique du Nord, 1950-2050

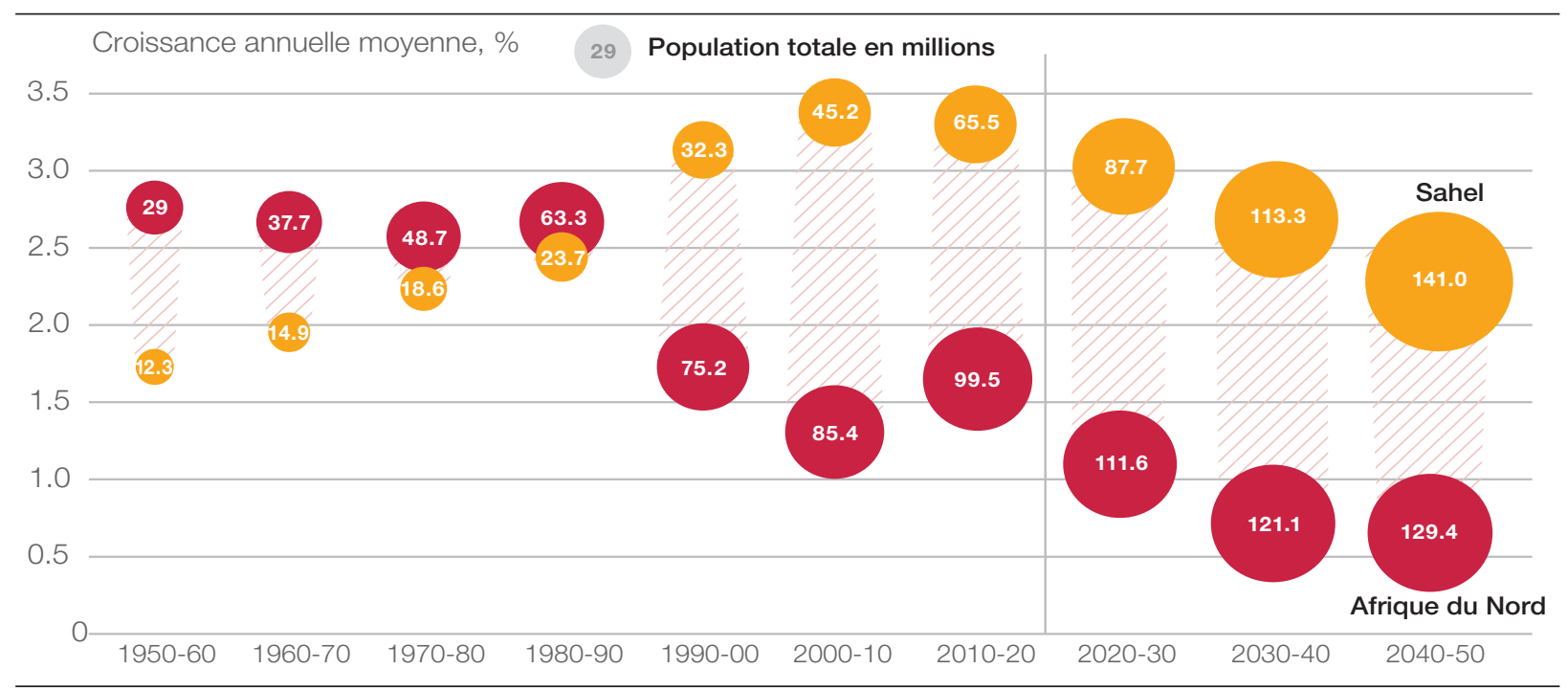

Note : Les taux de croissance annuelle moyens se réfèrent à la fin de la période d'observation. Projections basses pour le Sahel et moyennes pour l'Afrique du Nord.

Source : OCDE/CSAO (2014[3]). Mis à jour par l'auteur à partir de Nations Unies (2019[1]).

Graphique 19.

Projection du nombre d'habitants par pays, 1950-2050 (en millions d'habitants)

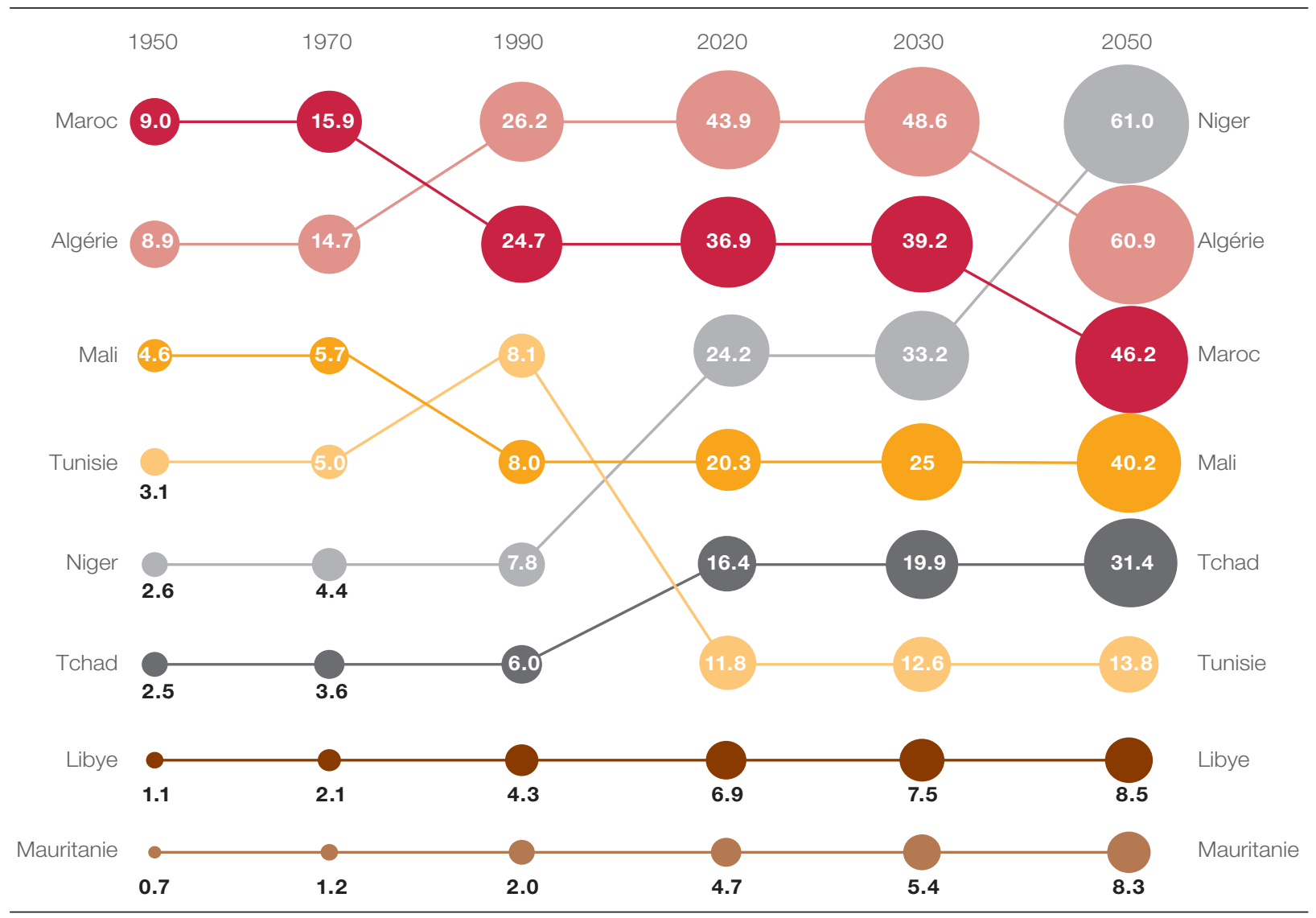

Note : Les taux de croissance annuelle moyens se réfèrent à la fin de la période d'observation. Projections basses pour le Sahel et moyennes pour l'Afrique du Nord.

Source : OCDE/CSAO (2014[3]). Mis à jour par l'auteur à partir de Nations Unies (2019[1]). 


\section{Perspectives}

Les pays d'Afrique du Nord et de l'Ouest sont résolument engagés dans le processus de transition démographique synonyme de chute de la mortalité, puis de la natalité. Au nord du Sahara, où le processus est le plus avancé, le nouvel équilibre démographique est marqué par une natalité plus forte que ce que le modèle théorique prédit, avec pour conséquence une croissance continue de la population. Au sud du Sahara, tous les pays ont connu une chute rapide de la mortalité suivie d'un recul de la natalité. Le décalage entre l'évolution des deux variables a contribué à un accroissement naturel spectaculaire en l'espace de quelques décennies. L'évolution des données les plus récentes suggère que la croissance annuelle moyenne de la population ralentira pour atteindre environ $2 \%$ en 2050 contre plus de $3 \%$ actuellement. Il est dès lors probable que tous les pays atteindront un nouvel équilibre démographique dans les prochaines décennies, y compris au Sahel.

La croissance démographique importante de la région s'accompagne d'une redistribution des populations en faveur des zones urbaines, qui progressent à la fois du fait de l'accroissement naturel et des migrations régionales. Au nord du Sahara, plus de 70 \% de la population vit désormais en ville et cette proportion devrait continuer à croître dans les prochaines décennies. Au sud du Sahara, où les villes croissent en nombre et en taille à un rythme élevé, près de la moitié des habitants sont urbains. L'urbanisation que connaît l'Afrique de l'Ouest est susceptible d'accélérer les changements sociaux, économiques et politiques qui favorisent la transition démographique.

Dans cette région, l'un des défis principaux est de parvenir à réduire les variations régionales observées dans les taux de fertilité, qui s'expliquent par un délai temporel entre zones urbaines et rurales du continent (Lerch, 2019[9]). Les techniques contraceptives, les nouvelles normes familiales et les nouvelles technologies se diffusent des villes aux campagnes à des vitesses différentes. Du fait de la mauvaise qualité des infrastructures, du faible niveau d'urbanisation et de l'absence de politiques publiques vigoureuses en matière de planning familial dans de nombreux pays ouest-africains, le déclin de la fertilité y est plus rapide en ville qu'en campagne contrairement à d'autres régions en développement où une convergence temporelle des taux est observée. Cette évolution conduit à une certaine déconnection entre la transition démographique des villes et celle des campagnes. 


\section{Références}

Banque mondiale (2021), Population Estimates and Projections, Banque mondiale. [13]

Bongaarts, J. (2017), «Africa's unique fertility transition », Population and Development Review, vol. 43/S1, pp. 39-58, http://dx.doi.org/10.1111/j.1728-4457.2016.00164.x. [8]

Corker, J. (2017), «Fertility and child mortality in urban West Africa: Leveraging geo-referenced data to move beyond the urban/rural dichotomy ", Population, Space and Place, Vol. 23/3, p. e2009, https://doi.org/10.1002/psp.2009. [12]

Doignon, Y. (2020), « Les transitions démographiques des pays méditerranéens depuis 1950 ", Géoconfluences. [7]

Flückiger, M. et M. Ludwig (2017), « Urbanization, fertility and child education in SubSaharan Africa », Economics Letters, vol. 157, pp. 97-102, http://dx.doi.org/10.1016/j. econlet.2017.05.024. [10]

Gries, T. et R. Grundmann (2018), «Fertility and modernization : the role of urbanization in developing countries ", Journal of International Development, vol. 30/3, pp. 493-506 http://dx.doi.org/10.1002/jid.3104. [11]

Lerch, M. (2019), «Fertility decline in urban and rural areas of developing countries », Population and Development Review, vol. 45/2, pp. 301-320, http://dx.doi.org/10.1111/padr.12220. [9]

Moriconi-Ebrard, F., D. Harre et P. Heinrigs (2016), L'urbanisation des pays de l'Afrique de l'Ouest 1950-2010: Africapolis I, mise à jour 2015, Cahiers de l'Afrique de l'Ouest, Éditions OCDE, Paris, https://dx.doi.org/10.1787/9789264252257-fr. [5]

Nations Unies (2019), World Population Prospects 2019, Nations Unies, Département des affaires économiques et sociales, Division de la population. [1]

OCDE/CSAO (2020), Dynamiques de l'urbanisation africaine 2020: Africapolis, une nouvelle géographie urbaine, Cahiers de l'Afrique de l'Ouest, Éditions OCDE, Paris, https://dx.doi. org/10.1787/481c7f49-fr. [2]

OCDE/CSAO (2014), Un atlas du Sahara-Sahel: Géographie, économie et insécurité, Cahiers de l'Afrique de l'Ouest, Éditions OCDE, Paris, https://dx.doi.org/10.1787/9789264222335-fr. [3]

OCDE/CSAO (2013), Peuplement, marché et sécurité alimentaire, Cahiers de l'Afrique de l'Ouest, Éditions OCDE, Paris, https://dx.doi.org/10.1787/9789264187412-fr. [4]

Our World in Data (2021), World Population Growth, https://ourworldindata.org/world-populationgrowth. [6] 


\section{Notes ouest-africaines}

\section{Urbanisation et démographie en Afrique du Nord et de l'Ouest, 1950-2020}

Cette note propose une analyse factuelle et rétrospective des relations entre urbanisation et démographie en Afrique de l'Ouest et du Nord. Elle montre que les pays de la région sont résolument engagés dans le processus de transition démographique. Au nord du Sahara, le nouvel équilibre démographique est marqué par une natalité plus forte que ce que le modèle théorique prédit, avec pour conséquence une croissance continue de la population. Plus de $70 \%$ de la population vit désormais en ville et cette proportion devrait continuer à croître dans les prochaines décennies. Au sud du Sahara, tous les pays ont connu une chute rapide de la mortalité suivie d'un recul de la natalité. Le décalage entre l'évolution des deux variables a contribué à un accroissement naturel spectaculaire en l'espace de quelques décennies. Cette croissance s'accompagne d'une redistribution des populations en faveur des zones urbaines, qui accueillent désormais près d'un habitant sur deux. L'urbanisation que connaît l'Afrique de l'Ouest est susceptible d'accélérer les changements sociaux, économiques et politiques qui favorisent la transition démographique. L'un des défis principaux est de parvenir à réduire les variations régionales observées dans les taux de fertilité, qui s'expliquent par un délai temporel entre zones urbaines et rurales du continent. 